Article

\title{
Dynamical Properties of Dark Energy Models in Fractal Universe
}

\author{
Muhammad Umair Shahzad ${ }^{1}$, Ayesha Iqbal ${ }^{2}$ and Abdul Jawad ${ }^{3 *(D)}$ \\ 1 CAMS, Business School, Faculty of Management Studies, University of Central Punjab, Lahore 54000, \\ Pakistan; m.u.shahzad@ucp.edu.pk \\ 2 Department of Mathematics, Govt. College University, Faisalabad 38000, Pakistan; \\ ayeshausmann@yahoo.com \\ 3 Department of Mathematics, COMSATS University Islamabad, Lahore-Campus, Lahore 54000, Pakistan \\ * Correspondence: abduljawad@cuilahore.edu.pk
}

Received: 30 July 2019; Accepted: 9 September 2019; Published: 16 September 2019

\begin{abstract}
In this paper, we consider the flat FRW spacetime filled with interacting dark energy and dark matter in fractal universe. We work with the three models of dark energy named as Tsallis, Renyi and Sharma-Mittal. We investigate different cosmological implications such as equation of state parameter, squared speed of sound, deceleration parameter, statefinder parameters, $\omega_{\text {eff }}-\omega_{\text {eff }}^{\prime}$ (where prime indicates the derivative with respect to $\ln a$, and $a$ is cosmic scale factor) plane and Om diagnostic. We explore these parameters graphically to study the evolving universe. We compare the consistency of dark energy models with the accelerating universe observational data. All three models are stable in fractal universe and support accelerated expansion of the universe.
\end{abstract}

Keywords: cosmological parameters; dark energy models; fractal universe

\section{Introduction}

The presence of networks of voids and structures in distribution of galaxies in space is one of the remarkable findings in the last two decades. The earlier pervasive examination of galaxy distribution with focus only on angular coordinates was unable to locate such high scale structures. Such findings refuse to accommodate the standard cosmological theories, in which homogeneity at large scale is of immense importance [1]. The existence of such a large scale at which the matter distribution approaches an average density is a matter of intense debate [2-4]. At small scales, it is well established that the distribution of galaxies is fractal, although its deviation from homogeneity is still debatable. Some statistical methods have been used to analyze the three dimensional data with some disagreements [3,4]. The fractal scenario is still resisted due to its incompatibility with the standard theories and isotropy of microwave back ground radiation $[5,6]$. The fractal distribution for matter was suggested in the framework of an expanding universe, the Friedmann cosmology [7].

A fractal is an identical and fundamental altering distribution of points at all scales, excluding its gravitational dynamics in framework of FRW solutions to general relativity [8,9]. The problem arises because of incompatibility of a fractal with the Cosmological Principle, which requires matter distribution to be isotropic and homogeneous [5]. In the case of non-analytical structure, such as fractal, all points are statistically equivalent, satisfying the conditional cosmological principle [8-10]. Systems whose effective dimensionality changes with scale may have fractal behavior. To understand the fractal effects, the present work is an effort to analyze cosmic acceleration under influence of fractal effects.

Many pieces of observational evidence such as different luminosity type Ia supernovae (SNIa) [11], Large Scale Structure [12] and Cosmic Microwave Background (CMB) radiation [13] strongly support the accelerated expansion of our universe. The candidates responsible for this expanding scenario 
provoked a new type of matter violating strong energy condition, i.e., $\rho+p<0$. Such exotic matters with negative energy density are named as Dark Energy (DE) [14]. One amongst the proposed candidates of DE appealing researchers the most is Holographic Dark energy (HDE). The idea behind this model is the Holographic principle, which emphasizes that for a physical system the number of degrees of freedom should be scaled by its bounding area instead of its volume $[15,16]$ constrained by infrared cutoff [17]. By using this idea, Li [18] recommended the following constraint on its energy density: $\rho_{d} \leq 3 C^{2} M_{p}^{2} / L^{2}$, where the equality sign holds only for saturated holographic bound. Here, $C^{2}$ is the dimensionless constant, $L$ denotes the IR-cutoff radius and $M_{p}^{2}=(8 \pi G)^{-1}$ is the reduced Plank mass. Li used three options for IR cutoffs, namely Hubble horizon, the future event horizon and the particle horizon. According to Li, only the future event horizon has the capability of accommodating the sufficient acceleration of universe. Fischler and Susskind proposed HDE models based on the cosmological state of Holographic principal [19]. The derivation of holographic energy density $\rho_{d}=3 C^{2} M_{p}^{2} / L^{2}$ is based on the entropy-area relationship $S \sim A \sim L^{2}$ of black holes. Here, $A=4 \pi L^{2}$ is area of horizon. A more general representation of HDE model with NO cutoff was presented Nojiri and Odinstov, who studied its cosmological and thermodynamical aspects [20,21].

Sheykhi [22] developed HDE model by using Hubble horizon as IR cutoff and believed that this model is capable of illustrating present state of universe by considering interaction of DE and Dark matter (DM). Hu and Ling examined the interacting HDE model and cosmological parameters through observational constraints [23]. In the low red-shift region, the HDE model supports the present observations. For a specific choice of interacting term, they attempted to reduce the cosmic coincidence problem by using different options for time rate of change of ratio of DM to HDE densities. By using a number of recent observational schemes, Ma et al. [24] observed big rip singularity for different parameters while exploring interacting and non-interacting HDE with DM. They found that HDE models are more compatible with observations as compared to $\Lambda$ CDM Model.

The DE and horizon entropy has an apparent influence on each other in thermodynamics. The exotic nature of space time and the Bekenstein entropy being non-extensive entropy measure, the generalized entropies, i.e., Tsallis and Renyi entropies, have been used to examine cosmological and gravitational phenomenon [25-28]. Amongst several generalized entropy formalisms to study cosmological phenomena, Tsallis and Renyi entropies develop more precise pictures of the universe. Another generalized entropy called the Sharma-Mittal measure [25] has been used to propose a new model of HDE [29]. In this model, the role of IR-cutoff is played by the Hubble horizon. It is stable and compatible with universe expansion history.

In the present work, we study the cosmological implications of HDE in fractal universe. We work in a flat FRW universe filled with interacting DM and DE. We use three different HDE models, namely Tsallis Holographic dark energy (THDE), Renyi Holographic dark energy (RHDE) and Sharma-Mittal Holographic dark energy (SMHDE), for this purpose. These models of HDE can also be generated from extended generalized form of EoS as presented by [21] and also presented in [30]. For analysis, we take help of EoS parameter, square speed of sound and deceleration parameter. Further, we make use of $\omega_{e f f}-\omega_{e f f}^{\prime}$, statefinders $\{r, s\}$ and Om diagnostics. In Section 2, the field equations under selected scenario and expressions for all the used HDE models are given. Section 3 contains discussion regarding deceleration parameter for the three HDE models in fractal universe. Similarly, Sections 4-8 contain the views about EoS parameter, square speed of sound, statefinder parameter, Om diagnostic and $\omega_{\text {eff }}-\omega_{\text {eff }}^{\prime}$, respectively. The last section comprises conclusion after detailed discussions of the previous sections.

\section{Field Equations with Solutions}

In a Fractal universe, time and space coordinates scale identically, i.e., $\left[x^{\mu}\right]=-1, \mu=0,1, \ldots, D-1$. The non-trivial measure (as in Lebesgue-Stieltjes integrals): $d^{D} x \rightarrow d \rho(x),[\rho]=-D \alpha \neq-D$ replaces the standard measure in action. Here, $\mathrm{D}$ is the topological dimension of the embedding space-time. During the evolution of the system, for a given time, a preserved fraction of states is 
represented roughly by the parameter $\alpha>0$. Such structures are obtainable by varying effective dimensionality of universe at different scales. This attribute can be acquired by introducing fractal action $[31,32]$. The general Boral probability measure $\rho$ is assumed as measure on Fractal set. Therefore, the metric-spacetime $M$ furnished with measure $\rho$ is represented by $(M, \rho)$ in $D$ dimensions. In this case, $\rho$ is continuous with $d \rho=d^{D} x v(x)$, some multidimensional Lebesgue measure, and $v$ is the weight function (fractal function). The total action of Einstein gravity in fractal spacetime can be given as

$$
S=S_{g}+S_{m}
$$

The gravitational part of action is

$$
S_{g}=M_{p}^{2} \int d^{D} x v(x) \sqrt{-g}\left(R-2 \Lambda-\omega \partial_{\mu} v \partial^{\mu} v\right),
$$

and

$$
S_{m}=\int d^{D} x v(x) \sqrt{-g} L_{m}
$$

is action of matter part minimally coupled with gravity [31-33]. Here, $M_{p}=(8 \pi G)^{-\frac{1}{2}}$ is the reduced Planck mass and $\omega$ represents fractal parameter, $R$ is the Ricci scalar.

In homogeneous, isotropic FLRW spacetime model, the Friedmann equations in a fractal universe can be obtained by taking variation of action with respect to metric tensor as given below

$$
H^{2}+\frac{k}{a^{2}}+H \frac{\dot{v}}{v}-\frac{\omega}{6} \dot{v}^{2}=\frac{1}{3}\left(\rho_{d}+\rho_{m}\right)+\frac{\Lambda}{3}
$$

Here, $\rho_{d}$ and $\rho_{m}$ are energy densities of DE and DM, respectively, $H=\frac{\dot{a}}{a}$ is Hubble parameter. $k$ is the curvature constant with $k=-1,0,1$ for open, flat and closed universe, respectively. We take time-like fractal profile $v=a^{-\gamma}$ with assumptions $\Lambda=0, k=0$ and $\gamma=$ constant. Now, the Equation (2) takes the following form

$$
H^{2}\left(1-\gamma-\frac{\omega}{6} v^{2} \gamma^{2}\right)=\frac{1}{3}\left(\rho_{d}+\rho_{m}\right)
$$

The energy conservation equation in Fractal universe is given as

$$
\dot{\rho}+(\rho+p)\left(3 H+\frac{\dot{v}}{v}\right)=0 .
$$

After substituting $v=a^{-\gamma}$, Equation (4) can be written as

$$
\dot{\rho}+H(\rho+p)(3-\gamma)=0 .
$$

We introduce the phenomenological term $Q$ to represent interaction between dark matter and dark energy model. Here, the decay rate should be proportional to the present value of Hubble parameter $H$ as determined by Supernova and $\mathrm{CMB}$ data. The interaction term $Q$ describes the energy flow between the two fluids. Observations from [34,35] give clear picture of interaction between DE and DM. The supernova data together with $\mathrm{CMB}$ and large scale structure [36] describe that such an interaction could be inferred from the expansion history of the universe. The relevant studies on the interaction between DE and DM have been carried out in [37-39]. The transition from matter domination to $\mathrm{DE}$ domination is given by an appropriate energy exchange rate. An interaction term having decay rate proportional to present value of Hubble parameter is assumed to be a good fit to the expansion history of the universe. The evidence for such interacting DE has also been provided by Baryon Oscillation Spectroscopic Survey (BOSS) [40]. Some authors [41] have also discussed various 
DE models in different frameworks and found interesting results. Amongst the different forms of $Q$ available in the literature, we choose the following form of $Q$ as in [42]

$$
Q=3 \xi H \rho_{m}
$$

Here, $\xi$ (may be positive or negative) is the interaction parameter. Now the conservation equation takes the following form

$$
\dot{\rho}_{d}+H\left(\rho_{d}+p_{d}\right)(3-\gamma)=-Q, \quad \rho_{m}+H\left(\rho_{m}\right)(3-\gamma)=Q,
$$

with $p_{m}=0$ being pressureless fluid. From Equation (7), we can easily find

$$
\rho_{m}=\rho_{0}(1+z)^{-(3 \tilde{\xi}+\gamma-3)} .
$$

Next, we discuss the energy densities for HDE models under consideration.

\subsection{THDE}

The Tsallis definition of entropy plays a vital role in the study of gravitational and cosmological systems. The Tsallis entropy content of system is the power law function of system area confirmed by the quantum gravity considerations. The horizon entropy of black hole can be modified as shown by Tsallis and Cirto [43].

$$
S_{\delta}=\gamma A^{\delta}
$$

Here, $\delta$ is the non additivity parameter [43] and $\gamma$ is unknown constant and $A=4 \pi L^{2}$ representing the area of horizon. The Bekenstein entropy is recoverable at the limit $\delta=1$ and $\gamma=\frac{1}{4 G}$ with $\hbar=k_{b}=c=1$. At this limit, the system is describable by ordinary distribution of probability. By using Equation (9) and relation $L^{3} \Lambda^{3} \leq\left(S_{\delta}\right)^{\frac{3}{4}}$ described by Cohen et al. [17], we get $\Lambda^{4} \leq \gamma(4 \pi)^{\delta} L^{2 \delta-4}$. This gives the following form to energy density of Tsallis holographic dark energy (THDE) [44].

$$
\rho_{d}=B H^{4-2 \delta} .
$$

By using Equations (3) and (7), we can calculate $\dot{H}$ and $p_{d}$ as

$$
\begin{aligned}
\dot{H}= & \frac{\rho_{0}(3 \xi+\gamma-3)(1+z)^{-(3 \xi+\gamma-3)} H-H^{3} \omega \gamma^{3}(1+z)^{2 \gamma}\left(\gamma^{2}\right)}{6 H\left(1-\gamma-\frac{\omega}{6}(1+z)^{2 \gamma} \gamma^{2}\right)-B(4-2 \delta) H^{3-2 \delta}}, \\
p_{d}= & 3 \xi \rho_{0}(1+z)^{-(3 \xi+\gamma-3)}-B H^{4-2 \delta}+\frac{B(4-2 \delta) H^{2-2 \delta}}{\gamma-3} \\
& \frac{\rho_{0}(3 \xi+\gamma-3)(1+z)^{-(3 \xi+\gamma-3)} H-H^{3} \omega \gamma^{3}(1+z)^{2 \gamma}\left(\gamma^{2}\right)}{6 H\left(1-\gamma-\frac{\omega}{6}(1+z)^{2 \gamma} \gamma^{2}\right)-B(4-2 \delta) H^{3-2 \delta}} .
\end{aligned}
$$

2.2. $R H D E$

Renyi $(S)$ and Tsallis $\left(S_{T}\right)$ are two well-known one-parameter generalized entropies defined as

$$
\begin{aligned}
S & =\frac{1}{\delta} \ln \sum_{i=1}^{W} P_{i}^{1-\delta}, \\
S_{T} & =\frac{1}{\delta} \sum_{i=1}^{W}\left(P_{i}^{1-\delta}-P_{i}\right) .
\end{aligned}
$$


Here, $\delta=1-Q$; combining the above one-parametric entropy measures with each other, we can get the following relation as in [45-48]

$$
S=\frac{1}{\delta} \ln \left(1+\delta S_{T}\right) .
$$

Using the above equation and assumption $\rho_{d} d V \propto T d S$, Renyi holographic dark energy (RHDE) can be defined as

$$
\rho_{d}=\frac{3 C^{2} H^{2}}{8 \pi\left(1+\frac{\delta \pi}{H^{2}}\right)} .
$$

The following expression for $\dot{H}$ and $p_{d}$ can be obtained by using Equations (3) and (7):

$$
\begin{gathered}
\dot{H}=\frac{\frac{\rho_{0}}{3}(3 \xi+\gamma-3)(1+z)^{-(3 \xi+\gamma-3)} H-H^{3}\left(\frac{\omega \nu^{2} \gamma^{3}}{3}\right)}{2 H\left(1-\gamma-\frac{\omega}{6} v^{2} \gamma^{2}\right)-\frac{C^{2}}{4 \pi} H^{3}\left(H^{2}+\delta \pi\right)^{-2}\left(H^{2}+2 \delta \pi\right)}, \\
p_{d}=\frac{-1}{H(3-\gamma)}\left(3 \xi H \rho_{0}(1+z)^{-(3 \xi+\gamma-3)}+\frac{3 C^{2} H^{3}(3-\gamma)}{8 \pi\left(1+\frac{\delta \pi}{H^{2}}\right)}+2 F H^{3}\left(H^{2}+2 \delta \pi\right)\right. \\
\left.\left(H^{2}+\delta \pi\right)^{-2} \frac{\frac{\rho_{0}}{3}(3 \xi+\gamma-3)(1+z)^{-(3 \xi+\gamma-3)} H-H^{3}\left(\frac{\omega \nu^{2} \gamma^{3}}{3}\right)}{2 H\left(1-\gamma-\frac{\omega}{6} v^{2} \gamma^{2}\right)-\frac{C^{2}}{4 \pi} H^{3}\left(H^{2}+\delta \pi\right)^{-2}\left(H^{2}+2 \delta \pi\right)}\right) .
\end{gathered}
$$

\subsection{SMHDE}

Another generalized entropy measure introduced by Sharma and Mittal defined as $[49,50]$

$$
S_{S M}=\frac{1}{1-r}\left(\left(\sum_{i=1}^{W} P_{i}^{1-\delta}\right)^{\frac{1-r}{\delta}}-1\right)
$$

Using Equations (13)-(15), one can easily write

$$
S_{S M}=\frac{1}{R}\left(\left(1+\delta S_{T}\right)^{\frac{R}{\delta}}-1\right),
$$

with $R=1-r$. As Bekenstein-Hawking entropy is supposed to be an appropriate candidate for Tsallis entropy, we can replace $S_{T}$ in Equation (20) by $S_{B}=\frac{A}{4}$ with $A$ being the horizon area and get

$$
S_{S M}=\frac{1}{R}\left(\left(1+\delta \frac{A}{4}\right)^{\frac{R}{\delta}}-1\right),
$$

Using Equation (21) and $\Lambda^{4} \propto \frac{S}{L^{4}}$ as described above, we approach SMHDE density given below

$$
\rho_{d}=\frac{3 C^{2} H^{4}}{8 \pi R}\left(\left(1+\frac{\pi \delta}{H^{2}}\right)^{\frac{R}{\delta}}-1\right)
$$

with $C^{2}$ being free parameter. With the help of Equations (3), (7) and (22), we derive the following expressions for $\dot{H}$ and $p_{d}$

$$
\begin{aligned}
\dot{H} & =\left(\frac{\rho_{0}}{3}(3 \xi+\gamma-3)(1+z)^{-(3 \xi+\gamma-3)} H-H^{3}\left(\frac{\omega v^{2} \gamma^{3}}{3}\right)\right)\left(2 H\left(1-\gamma-\frac{\omega}{6} v^{2} \gamma^{2}\right)\right. \\
& \left.-\frac{C^{2}}{8 \pi R}\left(4 H^{3}\left(1+\frac{\delta \pi}{H^{2}}\right)^{\beta}-1\right)-2 \delta \pi \beta H\left(1+\frac{\delta \pi}{H^{2}}\right)^{\beta-1}\right)^{-1}
\end{aligned}
$$




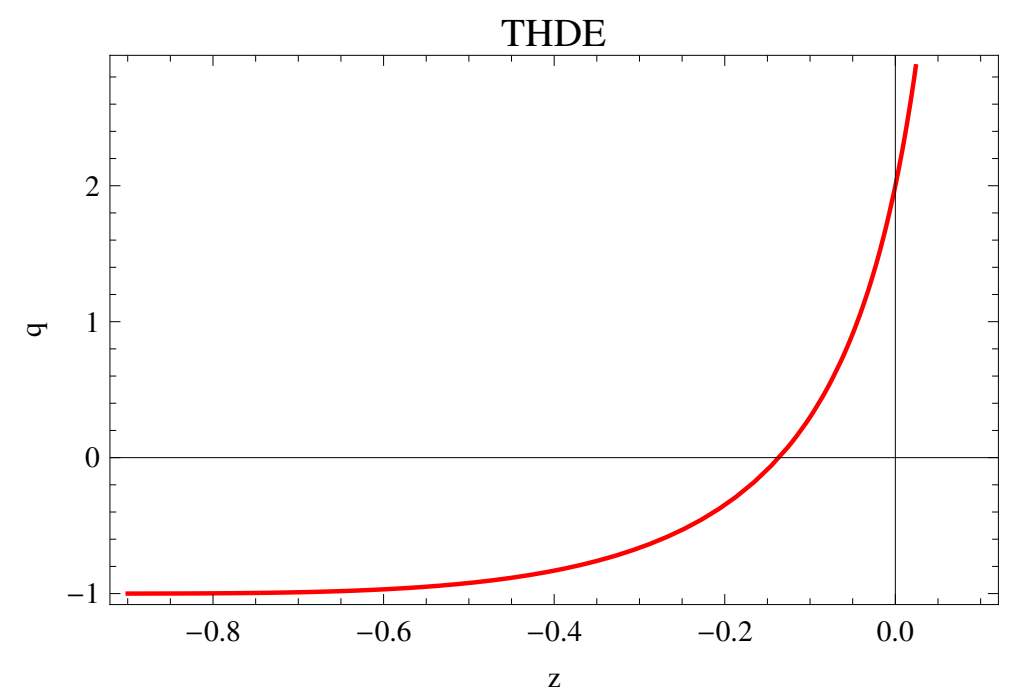

Figure 1. Deceleration parameter.

$$
\begin{aligned}
p_{d}= & \frac{1}{H(3-\gamma)}\left(-3 \xi H \rho_{0}(1+z)^{-(3 \xi+\gamma-3)}-\frac{3 C^{2} H^{5}(3-\gamma)}{8 \pi R}\left(\left(1+\frac{\delta \pi}{H^{2}}\right)^{\beta}-1\right)\right. \\
- & \left.\frac{3 C^{2}}{8 \pi R}\left(4 H^{3}\left(\left(1+\frac{\delta \pi}{H^{2}}\right)^{\beta}-1\right)-2 \delta \pi \beta H\left(1+\frac{\delta \pi}{H^{2}}\right)^{\beta}\right)\right)\left(\frac{\rho_{0}}{3}(3 \xi+\gamma-3)\right. \\
& \left.(1+z)^{-(3 \xi+\gamma-3)} H-H^{3}\left(\frac{\omega v^{2} \gamma^{3}}{3}\right)\right)\left(2 H\left(1-\gamma-\frac{\omega}{6} v^{2} \gamma^{2}\right)-\left(\frac{C^{2}}{8 \pi R}\right.\right. \\
& \left.\left.\left(4 H^{3}\left(1+\frac{\delta \pi}{H^{2}}\right)^{\beta}-1\right)\right)-2 \delta \pi \beta H\left(1+\frac{\delta \pi}{H^{2}}\right)^{\beta-1}\right)^{-1} \cdot
\end{aligned}
$$

\section{Deceleration Parameter}

To determine the cosmological acceleration of any cosmological model, the deceleration parameter plays a vital role. A negative value of deceleration parameter $q<0$ exhibit cosmic acceleration of model under discussion, while positive $q>0$ shows a decelerating universe. The deceleration parameter can be calculated as

$$
q=-1-\frac{\dot{H}}{H^{2}}
$$

\subsection{THDE}

In the case of THDE, by using the expression of $\dot{H}$ from Equation (11) in Equation (25), we get the following expression for deceleration parameter $q$

$$
q=-1-\frac{\rho_{0}(3 \xi+\gamma-3)(1+z)^{-(3 \xi+\gamma-3)} H-H^{3} \omega \gamma^{3}(1+z)^{2 \gamma}}{6 H^{3}\left(1-\gamma-\frac{\omega}{6}(1+z)^{2 \gamma} \gamma^{2}\right)-B(4-2 \delta) H^{5-2 \delta}}
$$

In Figure 1, we plot the variation of deceleration parameter with respect to redshift parameter with in the range $-0.9 \leq z \leq 0.1$. Here, we take $\omega=-0.9, \xi=-2, B=-1, \rho_{0}=1, \gamma=2, \alpha=1$ and $\delta=2$. From the plot, we see that the deceleration parameter remains negative before $z=-0.15$, exhibiting the cosmic acceleration. After $z=-0.15$, the curve representing $q$ becomes positive. For the redshift parameter $0.1 \leq z \leq-0.15, q$ represents decelerated phase and then for $-0.15 \leq z \leq-0.9, q$ exhibits accelerated expansion. From the curve, it is evident that the universe is evolving from early decelerated phase towards late accelerated phase. 


\section{2. $R H D E$}

The following expression of deceleration parameter for RHDE can be obtained by substituting value of $\dot{H}$ from Equation (17) into Equation (25)

$$
q=-1-\frac{\rho_{0}(3 \xi+\gamma-3)(1+z)^{-(3 \xi+\gamma-3)} H-H^{3} \omega \gamma^{3} v^{2}}{6 H^{3}\left(1-\gamma-\frac{\omega}{6} v^{2} \gamma^{2}\right)-2 F H^{5}\left(H^{2}+\delta \pi\right)^{-2}\left(H^{2}+2 \delta \pi\right)} .
$$

Figure 2 contains the plot of the expression for deceleration parameter versus redshift parameter $-0.9 \leq z \leq 0.1$ for RHDE. Here, we use same values of constants as discussed above. We observe that the deceleration parameter $q$ remains negative before $z=-0.14$ and becomes positive after $z=-0.14$. It represents accelerated universe before about $z=-0.14$ and decelerated phase of universe after $z=-0.14$. This means our universe is going through an accelerated phase of expansion.

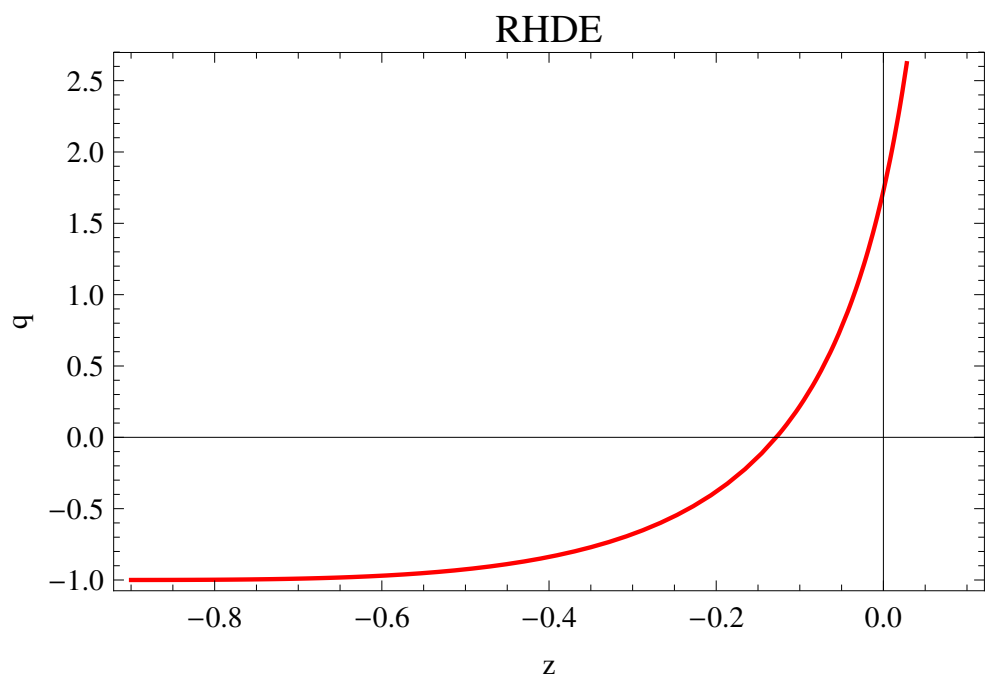

Figure 2. Deceleration parameter.

\section{3. $S M H D E$}

To get deceleration parameter expression in SMHDE, we replace $\dot{H}$ in Equation (25) by Equation (23) and achieve the following

$$
\begin{aligned}
q & =-1-H^{-2}\left(\frac{\rho_{0}}{3}(3 \xi+\gamma-3)(1+z)^{-(3 \xi+\gamma-3)} H-H^{3}\left(\frac{\omega v^{2} \gamma^{3}}{3}\right)\right)(2 H(1-\gamma \\
& \left.\left.-\frac{\omega}{6} v^{2} \gamma^{2}\right)-\left(\frac{C^{2}}{8 \pi R}\left(4 H^{3}\left(1+\frac{\delta \pi}{H^{2}}\right)^{\beta}-1\right)\right)-2 \delta \pi \beta H\left(1+\frac{\delta \pi}{H^{2}}\right)^{\beta-1}\right)^{-1} .
\end{aligned}
$$

The plot of the expression for deceleration parameter versus redshift parameter $-0.9 \leq z \leq 0.1$ for SMHDE is given in Figure 3. Under the same above-mentioned values for constants along with $C=1$ and $\beta=-800$, the behavior of deceleration parameter is identical with those in THDE and RHDE. It shows decelerated phase of the universe in the early epoch and eventually enters the era of accelerated expansion of the universe. 


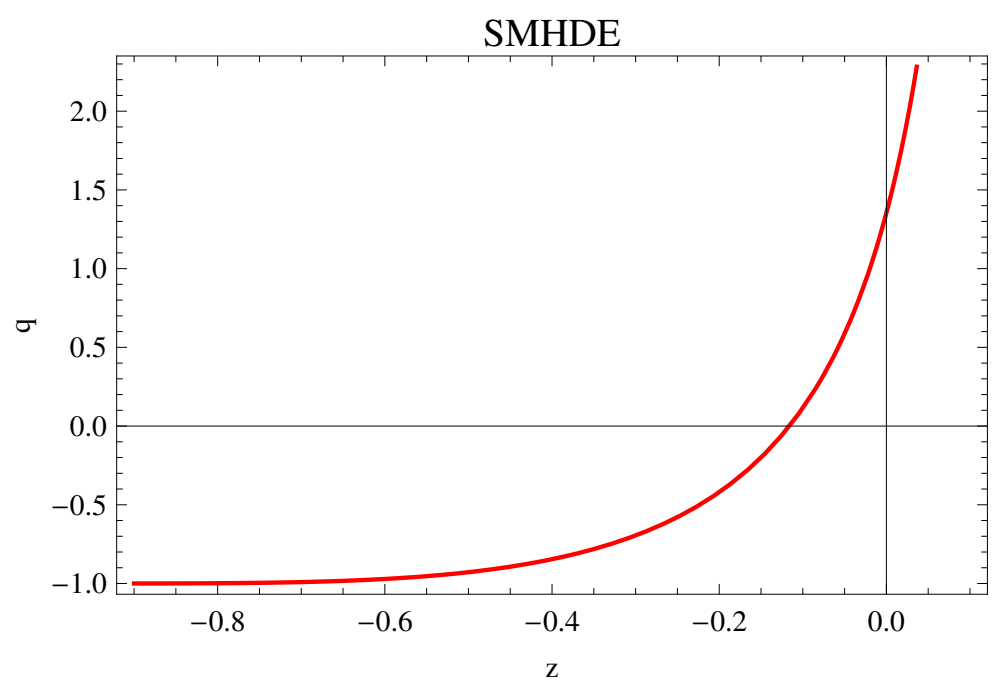

Figure 3. Deceleration parameter.

\section{Eos Parameter}

EoS parameter is mainly used for categorizing DE models, i.e., for non-relativistic matter we have $\omega_{\text {eff }}<0$. Likewise, for quintessence, we must have $-1<\omega_{\text {eff }}<\frac{-1}{3}$. The cosmological model is represented by $\omega_{\text {eff }}=-1$ and the phantom barrier holds for $\omega_{\text {eff }}<-1$. Similarly, $\omega_{\text {eff }}>-1$ corresponds to quintom behavior. To achieve the EoS parameter, we use the following equation

$$
\omega_{e f f}=\frac{p_{e f f}}{\rho_{e f f}} .
$$

\subsection{THDE}

By substituting values from Equations (10) and (12) into Equation (29), we get the following expression for square speed of sound.

$$
\begin{aligned}
\omega_{e f f}= & \left(\rho_{0}(1+z)^{-(3 \xi+\gamma-3)}+B H^{4-2 \delta}\right)^{-1}\left(\frac{3 \xi \rho_{0}(1+z)^{-(3 \xi+\gamma-3)}}{\gamma-3}-B H^{4-2 \delta}+(B\right. \\
& \left.\left.(4-2 \delta) \frac{H^{2-2 \delta}}{\gamma-3}\right) \frac{\rho_{0}(3 \xi+\gamma-3)(1+z)^{-(3 \xi+\gamma-3)} H-H^{3} \omega \gamma^{3}(1+z)^{2 \gamma}}{6 H\left(1-\gamma-\frac{\omega}{6}(1+z)^{2 \gamma} \gamma^{2}\right)-B(4-2 \delta) H^{3-2 \delta}}\right) .
\end{aligned}
$$

Figure 4 gives the plot of EoS parameter $\omega_{\text {eff }}$ for THDE for redshift parameter $-0.9 \leq z \leq-0.2$ with all the constants bearing same values as mentioned above. Following the classification described previously, we interpret the plot as below. The EoS parameter attains the value -1 from $z=-0.9$ to $z=-0.7$, showing that THDE is behaving as cosmological constant. Afterwards, it behaves as quintessence as it takes values within the range $-1<\omega_{\text {eff }}<-0.33$ when redshift parameter is in range $-0.7<z<-0.27$. For $z>-0.27$, THDE shows quintom behavior. 


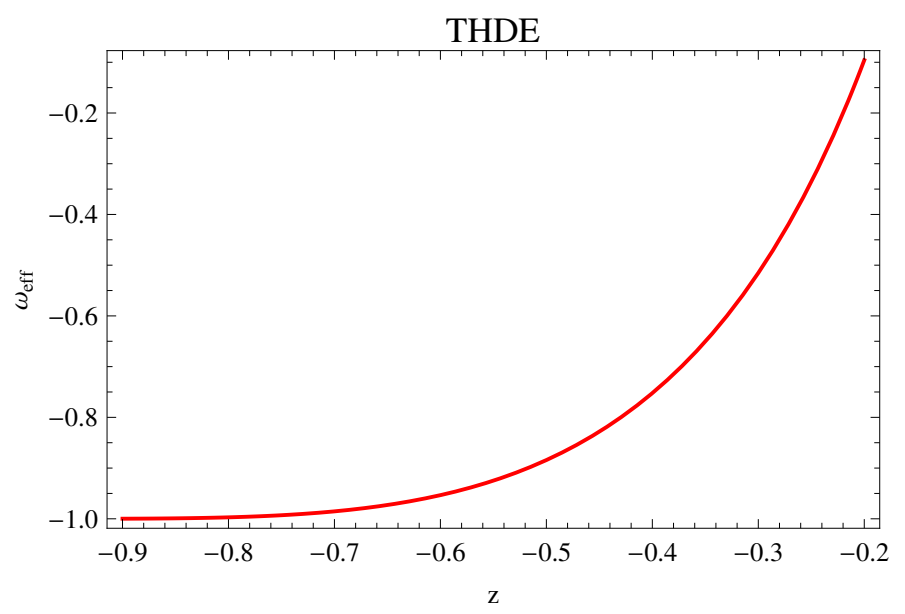

Figure 4. Eos parameter.

\subsection{RHDE}

To get an expression for EoS parameter in RHDE, we substitute values into Equation (29) from Equations (16) and (18)

$$
\begin{aligned}
\omega_{e f f}= & \frac{-1}{H(3-\gamma)}\left(3 \xi H \rho_{0}(1+z)^{-(3 \xi+\gamma-3)}+H(3-\gamma) F H^{4}\left(H^{2}+\delta \pi\right)^{-1}+\frac{2}{3} F H^{3}\right. \\
& \left(H^{2}+\delta \pi\right)^{-2}\left(H^{2}+2 \delta \pi\right)\left(\rho_{0}(3 \xi+\gamma-3)(1+z)^{-(3 \xi+\gamma-3)} H-\omega v^{2} \gamma^{2} H^{3}\right) \\
& \left.\left(2 H\left(1-\gamma-\frac{\omega}{6} v^{2} \gamma^{2}\right)-\frac{2}{3} F H^{3}\left(H^{2}+\delta \pi\right)^{-2}\left(H^{2}+2 \delta \pi\right)\right)^{-1}\right)\left(F H^{2}\right. \\
& \left.\left(H^{2}+\delta \pi\right)^{-1}+\rho_{0}(1+z)^{-(3 \xi+\gamma-3)}\right)^{-1} .
\end{aligned}
$$

The plot of EoS parameter $\omega_{\text {eff }}$ for RHDE against redshift parameter $-0.9 \leq z \leq-0.3$ is given by Figure 5. Here, all the constants have same values as mentioned above. Here, RHDE behaves as cosmological constant as the EoS parameter attains the value -1 from $z=-0.9$ to $z=-0.8$. Then, for redshift parameter $z>-0.8$, the curve lies in the range $-1<\omega_{\text {eff }}<-0.33$, showing the quintessence behavior.

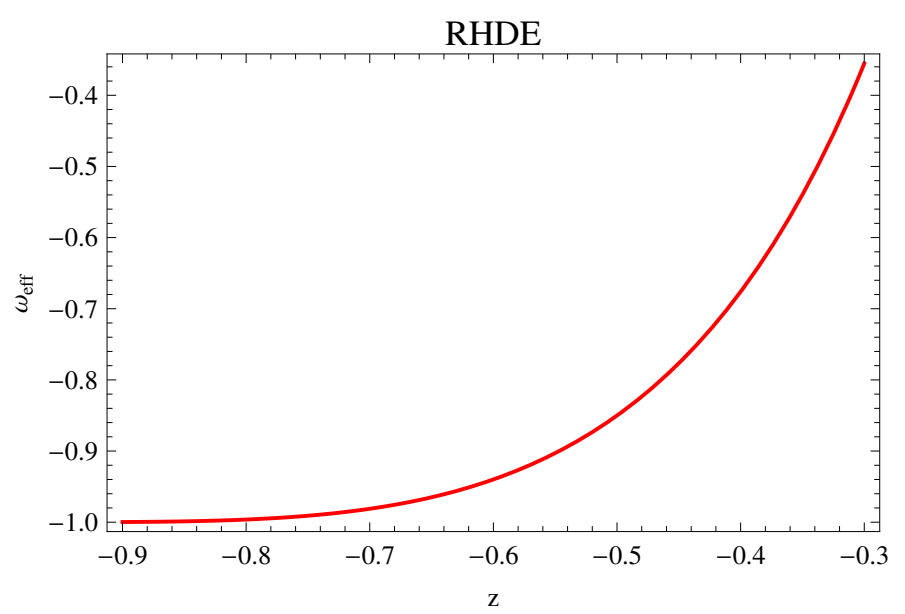

Figure 5. EoS parameter. 


\section{3. $S M H D E$}

To obtain the expression for EoS parameter in the case of SMHDE as given below we substitute values from Equations (22) and (24) into Equation (29)

$$
\begin{aligned}
\omega_{e f f}= & \frac{1}{H(3-\gamma)}\left(-3 \xi H \rho_{0}(1+z)^{-(3 \xi+\gamma-3)}-\frac{3 C^{2} H^{5}(3-\gamma)}{8 \pi R}\left(\left(1+\frac{\delta \pi}{H^{2}}\right)^{\beta}-1\right)\right. \\
- & \left.\frac{3 C^{2}}{8 \pi R}\left(4 H^{3}\left(\left(1+\frac{\delta \pi}{H^{2}}\right)^{\beta}-1\right)-2 \delta \pi \beta H\left(1+\frac{\delta \pi}{H^{2}}\right)^{\beta}\right)\right)\left(\frac{\rho_{0}}{3}(3 \xi+\gamma-3) H\right. \\
& \left.(1+z)^{-(3 \xi+\gamma-3)}-\frac{\omega v^{2} \gamma^{3} H^{3}}{3}\right)\left(2 H\left(1-\gamma-\frac{\omega v^{2} \gamma^{2}}{6}\right)-\frac{C^{2}}{8 \pi R}\left(4 H^{3}\left(1+\frac{\delta \pi}{H^{2}}\right)^{\beta}-1\right)\right. \\
- & \left.2 \delta \pi \beta H\left(1+\frac{\delta \pi}{H^{2}}\right)^{\beta-1}\right)^{-1}\left(\frac{3 C^{2} H^{4}}{8 \pi R}\left(\left(1+\frac{\delta \pi}{H^{2}}\right)^{\beta}-1\right)+\rho_{0}(1+z)^{-(3 \xi+\gamma-3)}\right)^{-1} .
\end{aligned}
$$

The graph for EoS parameter in case of SMHDE is given in Figure 6. Here, we use same previously described vales of constant for redshift parameter $-0.35 \leq z \leq-0.26$. For redshift parameter $-0.35 \leq z \leq-0.29$, SMHDE acts as quintessence and after $z=-0.29$ it shows quintom-like behavior.

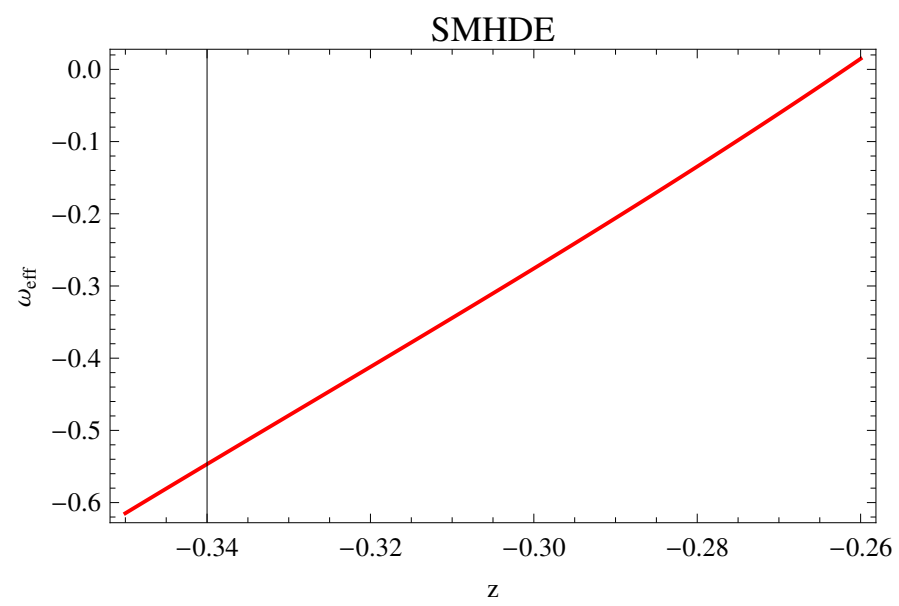

Figure 6. EoS parameter.

\section{Square Speed Of Sound}

For stability analysis of HDE models, we make use of the square speed of sound $C_{s}^{2}$. We calculate it as follows

$$
C_{s}^{2}=\frac{\partial p_{e f f}}{\partial \rho_{e f f}}
$$

Here, $p_{e f f}=p_{d}+p_{m}$ and $\rho_{e f f}=\rho_{d}+\rho_{m}$. For a HDE model to be stable, we must have $C_{s}^{2}>0$.

\subsection{THDE}

The expression for square speed of sound in THDE is as given below

$$
\begin{aligned}
C_{s}^{2}= & \left(\frac{3 \xi \rho_{0}(3 \xi+\gamma-3)(1+z)^{-(3 \xi+\gamma-3)}}{\gamma-3} H+\frac{B(4-2 \delta) H^{2-2 \delta}}{3(\gamma-3)}\right. \\
& \left(\left(\rho_{0}(3 \xi+\gamma-3)(1+z)^{-(3 \xi+\gamma-3)}-5 H^{2} \omega \gamma^{3}(1+z)^{2 \gamma}\right)\right. \\
& \frac{\rho_{0}(3 \xi+\gamma-3)(1+z)^{-(3 \xi+\gamma-3)} H-H^{3} \omega \gamma^{3}(1+z)^{2 \gamma}}{6 H\left(1-\gamma-\frac{\omega}{6}(1+z)^{2 \gamma} \gamma^{2}\right)-B(4-2 \delta) H^{3-2 \delta}}
\end{aligned}
$$




$$
\begin{aligned}
+ & \frac{\rho_{0}(3 \xi+\gamma-3)^{2}(1+z)^{-(3 \xi+\gamma-3)} H^{2}}{3}+\frac{2 \omega \gamma^{4} H^{4}(1+z)^{2 \gamma}}{3} \\
- & \left(2\left(1-\gamma-\frac{\omega}{6}(1+z)^{2 \gamma} \gamma^{2}\right)-\frac{B}{3}(4-2 \delta)(3-2 \delta) H^{2-2 \delta}\right) \\
& \left.\left(\frac{\rho_{0}(3 \xi+\gamma-3)(1+z)^{-(3 \xi+\gamma-3)} H-H^{3} \omega \gamma^{3}(1+z)^{2 \gamma}}{6 H\left(1-\gamma-\frac{\omega}{6}(1+z)^{2 \gamma} \gamma^{2}\right)-B(4-2 \delta) H^{3-2 \delta}}\right)^{2}\right) \\
& \left(2 H\left(1-\gamma-\frac{\omega}{6}(1+z)^{2 \gamma} \gamma^{2}\right)-\frac{B}{3}(4-2 \delta)(3-2 \delta) H^{2-2 \delta}\right)^{-1} \\
+ & \left(\frac{\rho_{0}(3 \xi+\gamma-3)(1+z)^{-(3 \xi+\gamma-3)} H-H^{3} \omega \gamma^{3}(1+z)^{2 \gamma}}{6 H\left(1-\gamma-\frac{\omega}{6}(1+z)^{2 \gamma} \gamma^{2}\right)-B(4-2 \delta) H^{3-2 \delta}}\right)^{2} \\
& \frac{B(4-2 \delta)(2-2 \delta)}{\gamma-3} H^{1-2 \delta}-B(4-2 \delta) H^{3-2 \delta} \\
& \left.\left(\frac{\rho_{0}(3 \xi+\gamma-3)(1+z)^{-(3 \xi+\gamma-3)} H-H^{3} \omega \gamma^{3}(1+z)^{2 \gamma}}{6 H\left(1-\gamma-\frac{\omega}{6}(1+z)^{2 \gamma} \gamma^{2}\right)-B(4-2 \delta) H^{3-2 \delta}}\right)\right) \\
& \left(\rho_{0}(3 \xi+\gamma-3)(1+z)^{-(3 \xi+\gamma-3)} H+B(4-2 \delta) H^{3-2 \delta}\right. \\
& \left.\frac{\rho_{0}(3 \xi+\gamma-3)(1+z)^{-(3 \xi+\gamma-3)} H-H^{3} \omega \gamma^{3}(1+z)^{2 \gamma}}{6 H\left(1-\gamma-\frac{\omega}{6}(1+z)^{2 \gamma} \gamma^{2}\right)-B(4-2 \delta) H^{3-2 \delta}}\right)^{-1} .
\end{aligned}
$$

In Figure 7, we plot the expression for $C_{s}^{2}$ versus redshift parameter $-0.7 \leq z \leq 0$ with same values of constants. The square speed of sound remains positive. This exhibits the stability of interacting THDE model in fractal universe.

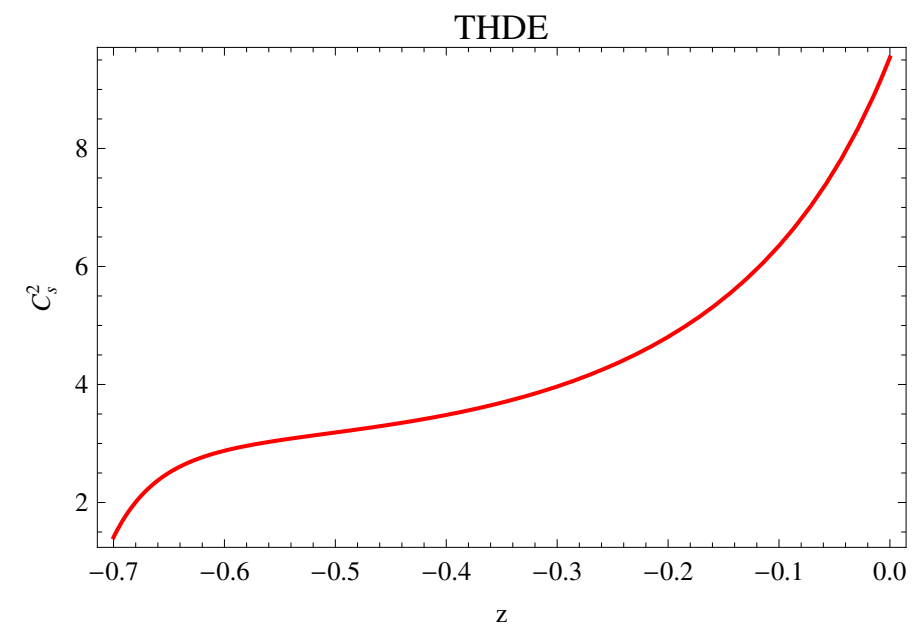

Figure 7. Square speed of sound.

\subsection{RHDE}

For RHDE, the square speed of sound has the following expression

$$
\begin{aligned}
C_{s}^{2}= & \frac{3 \xi \rho_{0}(3 \xi+\gamma-3)(1+z)^{-(3 \xi+\gamma-3)} H}{\gamma-3}-2 F H^{3}\left(H^{2}+\delta \pi\right)^{-2}\left(H^{2}+2 \delta \pi\right) \\
& \left(\frac{\frac{\rho_{0}}{3}(3 \xi+\gamma-3)(1+z)^{-(3 \xi+\gamma-3)} H-H^{3}\left(\frac{\omega \nu^{2} \gamma^{3}}{3}\right)}{2 H\left(1-\gamma-\frac{\omega}{6} v^{2} \gamma^{2}\right)-\frac{C^{2}}{4 \pi} H^{3}\left(H^{2}+\delta \pi\right)^{-2}\left(H^{2}+2 \delta \pi\right)}\right)+\frac{1}{\gamma-3} \\
& \left(H^{-1}\left(2 H\left(1-\gamma-\frac{\omega}{6} v^{2} \gamma^{2}\right)-\frac{2}{3} F H^{3}\left(H^{2}+\delta \pi\right)^{-2}\left(H^{2}+2 \delta \pi\right)\right)^{-1}\right.
\end{aligned}
$$




$$
\begin{aligned}
& 2 F H^{3}\left(H^{2}+\delta \pi\right)^{-2}\left(H^{2}+2 \delta \pi\right)\left(\frac{\rho_{0}}{3}(3 \xi+\gamma-3)^{2} H^{2}(1+z)^{-(3 \xi+\gamma-3)}\right. \\
& -\frac{2 \omega}{3} H^{4} \gamma^{4} v^{2}+2\left(\frac{\frac{\rho_{0}}{3}(3 \xi+\gamma-3)(1+z)^{-(3 \xi+\gamma-3)} H-H^{3}\left(\frac{\omega v^{2} \gamma^{3}}{3}\right)}{2 H\left(1-\gamma-\frac{\omega}{6} v^{2} \gamma^{2}\right)-\frac{C^{2}}{4 \pi} H^{3}\left(H^{2}+\delta \pi\right)^{-2}\left(H^{2}+2 \delta \pi\right)}\right)^{2} \\
& \left(F H^{2}\left(H^{2}+\delta \pi\right)^{-2}\left(H^{2}+2 \delta \pi\right)-\frac{4}{3} F H^{4}\left(H^{2}+\delta \pi\right)^{-3}\left(H^{2}+2 \delta \pi\right)\right. \\
& \left.+\frac{2}{3} F H^{4}\left(H^{2}+\delta \pi\right)^{-2}-\left(1-\gamma-\frac{\omega}{6} v^{2} \gamma^{2}\right)\right) \\
& -\left(\frac{\frac{\rho_{0}}{3}(3 \xi+\gamma-3)(1+z)^{-(3 \tilde{\xi}+\gamma-3)} H-H^{3}\left(\frac{\omega \nu^{2} \gamma^{3}}{3}\right)}{2 H\left(1-\gamma-\frac{\omega}{6} v^{2} \gamma^{2}\right)-\frac{C^{2}}{4 \pi} H^{3}\left(H^{2}+\delta \pi\right)^{-2}\left(H^{2}+2 \delta \pi\right)}\right) \\
& \left.\left(\frac{2}{3} H^{2} \omega \gamma^{3} v^{2}-H^{2} \omega \gamma^{3} v^{2}-\frac{\rho_{0}}{3}(3 \xi+\gamma-3)^{2}(1+z)^{-(3 \xi+\gamma-3)}\right)\right) \\
& +2 F\left(\frac{\frac{\rho_{0}}{3}(3 \xi+\gamma-3)(1+z)^{-(3 \xi+\gamma-3)} H-H^{3}\left(\frac{\omega \nu^{2} \gamma^{3}}{3}\right)}{2} H\left(1-\gamma-\frac{\omega}{6} v^{2} \gamma^{2}\right)\right. \\
& \left.-\frac{C^{2}}{4 \pi} H^{3}\left(H^{2}+\delta \pi\right)^{-2}\left(H^{2}+2 \delta \pi\right)\right)^{2}\left(3 H^{2}\left(H^{2}+\delta \pi\right)^{-2}\left(H^{2}+2 \delta \pi\right)-4 H^{4}\right. \\
& \left.\left(H^{2}+\delta \pi\right)^{-3}\left(H^{2}+2 \delta \pi\right)+2 H^{4}\left(H^{2}+\delta \pi\right)^{-2}\right)-\left(H^{2}+\delta \pi\right)^{-2}\left(H^{2}+2 \delta \pi\right) \\
& \left.2 F H\left(\frac{\frac{\rho_{0}}{3}(3 \xi+\gamma-3)(1+z)^{-(3 \xi+\gamma-3)} H-H^{3}\left(\frac{\omega v^{2} \gamma^{3}}{3}\right)}{2 H\left(1-\gamma-\frac{\omega}{6} v^{2} \gamma^{2}\right)-\frac{C^{2}}{4 \pi} H^{3}\left(H^{2}+\delta \pi\right)^{-2}\left(H^{2}+2 \delta \pi\right)}\right)^{2}\right) .
\end{aligned}
$$

The square speed of sound for RHDE is given in Figure 8 against the redshift parameter. Here, we take $\xi=2, \alpha=1$ and the other constants are same as described above. It is evident from plot that square speed of sound remains positive for $-0.46 \leq z \leq-0.454$. Hence, the RHDE model in fractal universe is stable.

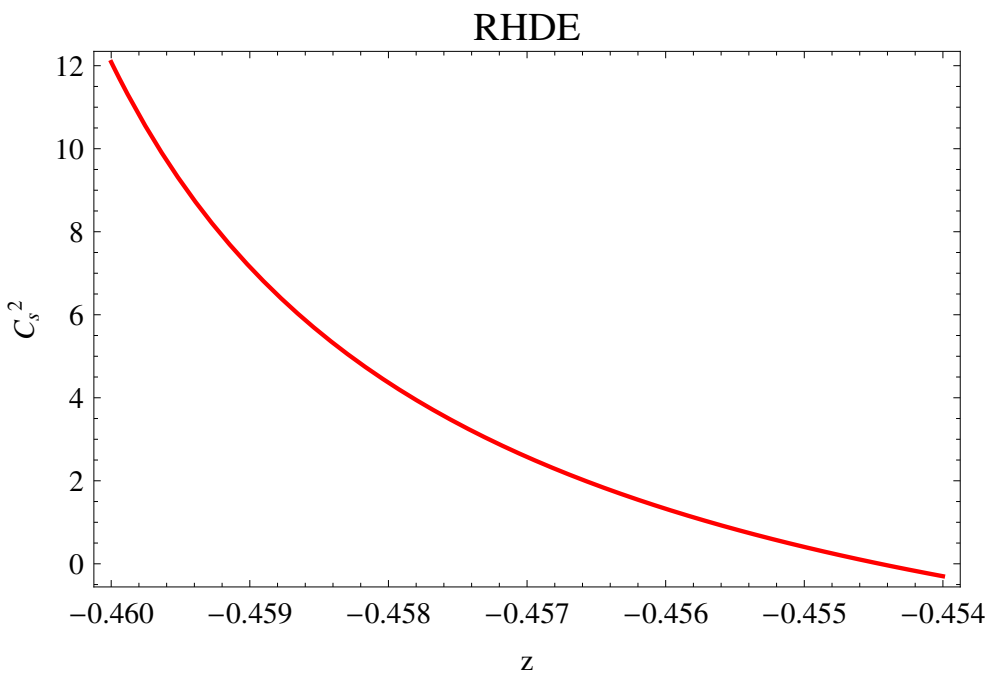

Figure 8. Square speed of sound.

\subsection{SMHDE}

The square speed of sound has the following expression in the case of SMHDE:

$$
C_{s}^{2}=\frac{1}{3-\gamma}\left(-3 \xi \rho_{0}(3 \xi+\gamma-3)(1+z)^{-(3 \xi+\gamma-3)}-H^{-1} \frac{3 C^{2}}{8 \pi R}\left(12 H^{2}\right.\right.
$$




$$
\begin{aligned}
& \left(\left(1+\frac{\delta \pi}{H^{2}}\right)^{\beta}-1\right)-10 \delta \pi \beta\left(1+\frac{\delta \pi}{H^{2}}\right)^{\beta-1}+4 \delta^{2} \pi^{2} \beta(\beta-1)\left(1+\frac{\delta \pi}{H^{2}}\right)^{\beta-1} \\
& \left.H^{-2}\right)\left(( \frac { \rho _ { 0 } } { 3 } ( 3 \xi + \gamma - 3 ) ( 1 + z ) ^ { - ( 3 \xi + \gamma - 3 ) } H - H ^ { 3 } ( \frac { \omega v ^ { 2 } \gamma ^ { 3 } } { 3 } ) ) \left(\left(1-\gamma-\frac{\omega}{6} v^{2} \gamma^{2}\right)\right.\right. \\
& \left.\left.2 H-\frac{C^{2}}{8 \pi R}\left(4 H^{3}\left(1+\frac{\delta \pi}{H^{2}}\right)^{\beta}-1\right)-2 \delta \pi \beta H\left(1+\frac{\delta \pi}{H^{2}}\right)^{\beta-1}\right)^{-1}\right)^{3} \\
& +\frac{3 C^{2}}{8 \pi R}\left(4 H^{3}\left(\left(1+\frac{\delta \pi}{H^{2}}\right)^{\beta}-1\right)-2 \delta \beta H\left(1+\frac{\delta \pi}{H^{2}}\right)^{\beta-1}\right)\left(2 H\left(1-\gamma-\frac{\omega}{6} \gamma^{2} v^{2}\right)\right. \\
& \left.-\frac{C^{2}}{8 \pi R}\left(4 H^{3}\left(\left(1+\frac{\delta \pi}{H^{2}}\right)^{\beta}-1\right)-2 \delta \beta H\left(1+\frac{\delta \pi}{H^{2}}\right)^{\beta-1}\right)\right)^{-1}\left(\frac{2}{3} \omega \gamma^{4} v^{2} H^{4}\right. \\
& +\frac{1}{3}\left(\rho _ { 0 } ( 3 \xi + \gamma - 3 ) ( 1 + z ) ^ { - ( 3 \xi + \gamma - 3 ) } \left(\left(\frac{\rho_{0}}{3}(3 \xi+\gamma-3)(1+z)^{-(3 \xi+\gamma-3)} H\right.\right.\right. \\
& \left.-H^{3} \frac{\omega v^{2} \gamma^{3}}{3}\right)\left(2 H\left(1-\gamma-\frac{\omega}{6} v^{2} \gamma^{2}\right)-\frac{C^{2}}{8 \pi R}\left(4 H^{3}\left(1+\frac{\delta \pi}{H^{2}}\right)^{\beta}-1\right)-2 \delta \pi \beta H\right. \\
& \left.\left.\left.\left(1+\frac{\delta \pi}{H^{2}}\right)^{\beta-1}\right)^{-1}\right)+\rho_{0}(3 \xi+\gamma-3)^{2} H^{2}(1+z)^{-(3 \xi+\gamma-3)}\right)-2\left(1-\gamma-\frac{\omega}{6} \gamma^{2} v^{2}\right) \\
& -\frac{C^{2}}{8 \pi R}\left(\frac{\rho_{0}}{3}(3 \xi+\gamma-3)(1+z)^{-(3 \xi+\gamma-3)} H-H^{3}\left(\frac{\omega v^{2} \gamma^{3}}{3}\right)\right)\left(2 H\left(1-\gamma-\frac{\omega}{6} v^{2} \gamma^{2}\right)\right. \\
& \left.-\frac{C^{2}}{8 \pi R}\left(4 H^{3}\left(1+\frac{\delta \pi}{H^{2}}\right)^{\beta}-1\right)-2 \delta \pi \beta H\left(1+\frac{\delta \pi}{H^{2}}\right)^{\beta-1}\right)^{-1}\left(12 H^{2}\left(\left(1+\frac{\delta \pi}{H^{2}}\right)^{\beta}-1\right)\right. \\
& \left.-10 \delta \pi \beta\left(1+\frac{\delta \pi}{H^{2}}\right)^{\beta-1}+4 \delta^{2} \pi^{2} \beta(\beta-1)\left(1+\frac{\delta \pi}{H^{2}}\right)^{\beta-1} H^{-2}\right)\left(\left(\frac{\rho_{0}}{3}(3 \xi+\gamma-3)\right.\right. \\
& \left.(1+z)^{-(3 \xi+\gamma-3)} H-H^{3} \frac{\omega v^{2} \gamma^{3}}{3}\right)\left(2 H\left(1-\gamma-\frac{\omega}{6} v^{2} \gamma^{2}\right)-\frac{C^{2}}{8 \pi R}\left(4 H^{3}\left(1+\frac{\delta \pi}{H^{2}}\right)^{\beta}\right.\right. \\
& \text { - 1) } \left.\left.-2 \delta \pi \beta H\left(1+\frac{\delta \pi}{H^{2}}\right)^{\beta-1}\right)^{-1}\right)^{2}-\frac{5}{3} \omega \gamma^{3} v^{2} H^{2}\left(\frac{\rho_{0}}{3}(3 \xi+\gamma-3)(1+z)^{-(3 \xi+\gamma-3)} H\right. \\
& \text { - } \left.H^{3}\left(\frac{\omega v^{2} \gamma^{3}}{3}\right)\right)\left(2 H\left(1-\gamma-\frac{\omega}{6} v^{2} \gamma^{2}\right)-\frac{C^{2}}{8 \pi R}\left(4 H^{3}\left(1+\frac{\delta \pi}{H^{2}}\right)^{\beta}-1\right)-2 \delta \pi \beta H\right. \\
& \left.\left.\left(1+\frac{\delta \pi}{H^{2}}\right)^{\beta-1}\right)^{-1}\right)+\left(H^{-2}-3+\gamma\right) \frac{3 C^{2}}{8 \pi R}\left(4 H^{3}\left(\left(1+\frac{\delta \pi}{H^{2}}\right)^{\beta}-1\right)-2 \delta \beta H\right. \\
& \left.\left(1+\frac{\delta \pi}{H^{2}}\right)^{\beta-1}\right)\left(\frac{\rho_{0}}{3}(3 \xi+\gamma-3)(1+z)^{-(3 \xi+\gamma-3)} H-H^{3}\left(\frac{\omega v^{2} \gamma^{3}}{3}\right)\right)(2 H \\
& \left.\left.\left(1-\gamma-\frac{\omega}{6} v^{2} \gamma^{2}\right)-\frac{C^{2}}{8 \pi R}\left(4 H^{3}\left(1+\frac{\delta \pi}{H^{2}}\right)^{\beta}-1\right)-2 \delta \pi \beta H\left(1+\frac{\delta \pi}{H^{2}}\right)^{\beta-1}\right)^{-1}\right) \\
& \left(\frac { 3 C ^ { 2 } } { 8 \pi R } ( 4 H ^ { 3 } ( ( 1 + \frac { \delta \pi } { H ^ { 2 } } ) ^ { \beta } - 1 ) - 2 \delta \beta H ( 1 + \frac { \delta \pi } { H ^ { 2 } } ) ^ { \beta - 1 } ) \left(\frac{\rho_{0}}{3}(3 \xi+\gamma-3)\right.\right. \\
& \left.(1+z)^{-(3 \xi+\gamma-3)} H-H^{3}\left(\frac{\omega v^{2} \gamma^{3}}{3}\right)\right)\left(2 H\left(1-\gamma-\frac{\omega}{6} v^{2} \gamma^{2}\right)-\frac{C^{2}}{8 \pi R}\left(4 H^{3}\right.\right. \\
& \left.\left.\left.\left(1+\frac{\delta \pi}{H^{2}}\right)^{\beta}-1\right)-2 \delta \pi \beta H\left(1+\frac{\delta \pi}{H^{2}}\right)^{\beta-1}\right)^{-1}\right)^{-1} .
\end{aligned}
$$

Figure 9 contains the plot of the square speed of sound for SMHDE, with all values of constants as mentioned above and $\xi$ as in the previous plot with $\beta=-800$. Here, the trajectory shows the stability of SMHDE model from $z=-0.8$ to $z=-0.5$. 


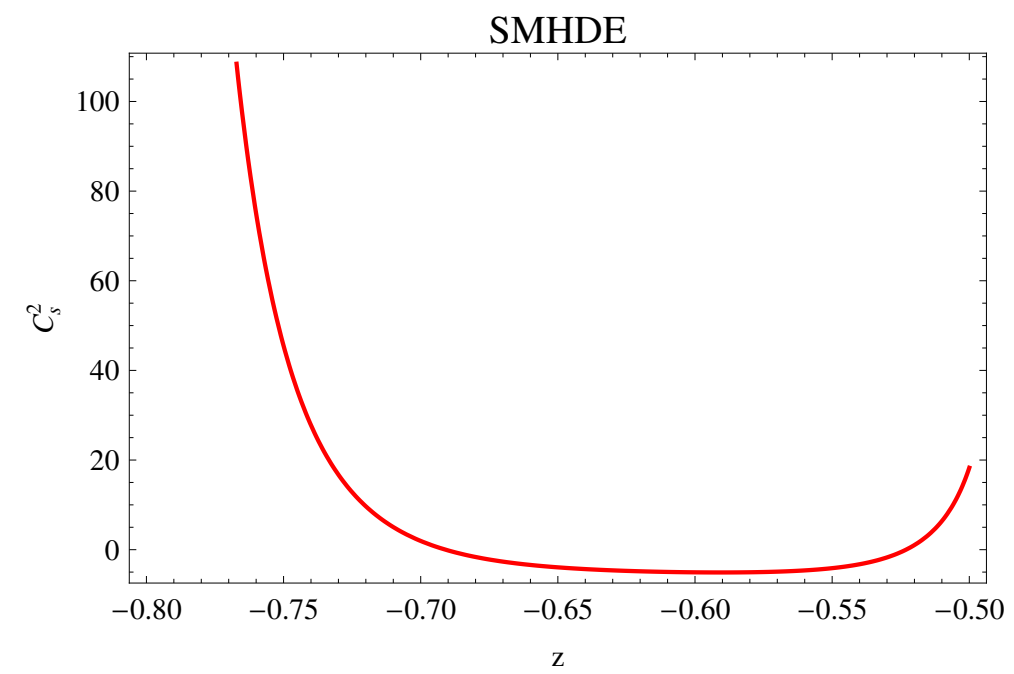

Figure 9. Square speed of sound.

\section{Statefinder Parameters}

To obtain a diagnostic tool involving higher derivative of scale factor, Sahni et al. [51] introduced the state finder pair $\{r, s\}$. It is a geometrical diagnostic being depending on scale factor. Mainly, it is used to recognize DE models. Distinct evolutionary trajectories are shown in $\{r, s\}$ plane by different DE models. Trajectories in s-r plane relevant to different cosmological models show qualitatively different behaviors. By using statefinder diagrams, we can explicitly diagnose the spatial curvature contribution in different DE models. This statefinder parameter $\{r, s\}$ in terms of Hubble parameter can be defined as

$$
r=1+3 \frac{\dot{H}}{H^{2}}+\frac{\ddot{H}}{H^{3}}
$$

and

$$
s=-\frac{3 H \dot{H}+\ddot{H}}{3 H\left(2 \dot{H}+3 H^{2}\right)} .
$$

\subsection{THDE}

By using Equation (11) in Equations (37) and (38), we get the following expressions for $r$ and $s$ :

$$
\begin{aligned}
r= & 1+\frac{3}{H^{2}} \frac{\rho_{0}(3 \xi+\gamma-3)(1+z)^{-(3 \xi+\gamma-3)} H-H^{3} \omega \gamma^{3}(1+z)^{2 \gamma}}{6 H\left(1-\gamma-\frac{\omega}{6}(1+z)^{2 \gamma} \gamma^{2}\right)-B(4-2 \delta) H^{3-2 \delta}} \\
+ & \frac{1}{H^{3}}\left(\left(\left(\frac{\rho_{0}}{3}(3 \xi+\gamma-3)(1+z)^{-(3 \xi+\gamma-3)}-\frac{5 H^{2} \omega \gamma^{3}(1+z)^{2 \gamma}}{3}\right)\right.\right. \\
& \frac{\rho_{0}(3 \xi+\gamma-3)(1+z)^{-(3 \xi+\gamma-3)} H-H^{3} \omega \gamma^{3}(1+z)^{2 \gamma}}{6 H\left(1-\gamma-\frac{\omega}{6}(1+z)^{2 \gamma} \gamma^{2}\right)-B(4-2 \delta) H^{3-2 \delta}}+\frac{\rho_{0}}{3}(3 \xi+\gamma-3) \\
& (1+z)^{-(3 \xi+\gamma-3)} H^{2}+\frac{2 \omega \gamma^{4}}{3}(1+z)^{2 \gamma}-\left(2 \left(1-\gamma-\frac{\omega}{6}(1+z)^{2 \gamma} \gamma^{2}-\frac{B}{3}(4-2 \delta)\right.\right. \\
& \left.\left.(3-2 \delta) H^{2-2 \delta}\right)\right)\left(\frac{\rho_{0}(3 \xi+\gamma-3)(1+z)^{-(3 \xi+\gamma-3)} H-H^{3} \omega \gamma^{3}(1+z)^{2 \gamma}}{6 H\left(1-\gamma-\frac{\omega}{6}(1+z)^{2 \gamma} \gamma^{2}\right)-B(4-2 \delta) H^{3-2 \delta}}\right) \\
& \left(2\left(1-\gamma-\frac{\omega}{6}(1+z)^{2 \gamma} \gamma^{2}-\frac{B}{3}(4-2 \delta)(3-2 \delta) H^{2-2 \delta}\right)^{-1}\right) \\
s= & \frac{1}{3}\left(\frac{3}{H^{2}} \frac{\rho_{0}(3 \xi+\gamma-3)(1+z)^{-(3 \xi+\gamma-3)} H-H^{3} \omega \gamma^{3}(1+z)^{2 \gamma}}{6 H\left(1-\gamma-\frac{\omega}{6}(1+z)^{2 \gamma} \gamma^{2}\right)-B(4-2 \delta) H^{3-2 \delta}}\right.
\end{aligned}
$$




$$
\begin{aligned}
+ & \frac{1}{H^{3}}\left(\left(\left(\frac{\rho_{0}}{3}(3 \xi+\gamma-3)(1+z)^{-(3 \xi+\gamma-3)}-\frac{5 H^{2} \omega \gamma^{3}(1+z)^{2 \gamma}}{3}\right)\right.\right. \\
& \frac{\rho_{0}(3 \xi+\gamma-3)(1+z)^{-(3 \xi+\gamma-3)} H-H^{3} \omega \gamma^{3}(1+z)^{2 \gamma}}{6 H\left(1-\gamma-\frac{\omega}{6}(1+z)^{2 \gamma} \gamma^{2}\right)-B(4-2 \delta) H^{3-2 \delta}}+\frac{\rho_{0}}{3}(3 \xi+\gamma-3) \\
& (1+z)^{-(3 \xi+\gamma-3)} H^{2}+\frac{2 \omega \gamma^{4}}{3}(1+z)^{2 \gamma}-\left(2 \left(1-\gamma-\frac{\omega}{6}(1+z)^{2 \gamma} \gamma^{2}-\frac{B}{3}(4-2 \delta)\right.\right. \\
& \left.\left.(3-2 \delta) H^{2-2 \delta}\right)\right)\left(\frac{\rho_{0}(3 \xi+\gamma-3)(1+z)^{-(3 \xi+\gamma-3)} H-H^{3} \omega \gamma^{3}(1+z)^{2 \gamma}}{6 H\left(1-\gamma-\frac{\omega}{6}(1+z)^{2 \gamma} \gamma^{2}\right)-B(4-2 \delta) H^{3-2 \delta}}\right) \\
& \left.\left(2\left(1-\gamma-\frac{\omega}{6}(1+z)^{2 \gamma} \gamma^{2}-\frac{B}{3}(4-2 \delta)(3-2 \delta) H^{2-2 \delta}\right)^{-1}\right)\right)(-1 \\
- & \left.\frac{\rho_{0}(3 \xi+\gamma-3)(1+z)^{-(3 \xi+\gamma-3)} H-H^{3} \omega \gamma^{3}(1+z)^{2 \gamma}}{6 H^{3}\left(1-\gamma-\frac{\omega}{6}(1+z)^{2 \gamma} \gamma^{2}\right)-B(4-2 \delta) H^{5-2 \delta}}\right)^{-1} .
\end{aligned}
$$

Figure 10 shows variation of statefinder parameter $s$ against $r$ in the case of THDE. It is evident from the plot that we have $r=1$ for $s=0$. This corresponds to the standard $\Lambda \mathrm{CDM}$ cosmological model for accelerating universe.

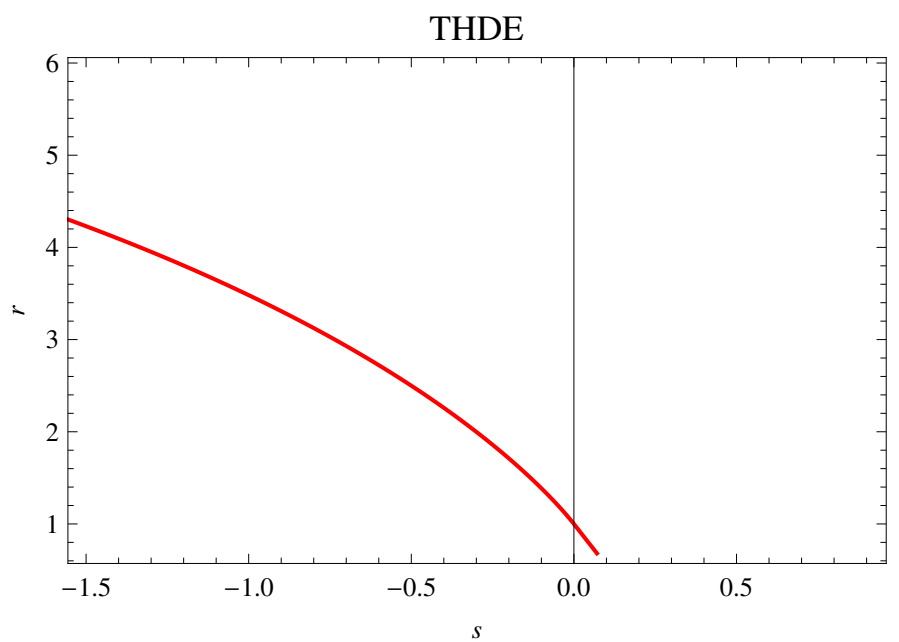

Figure 10. Statefinder parameter.

\section{2. $R H D E$}

In the case of RHDE, Equations (37) and (38) can be modified using Equation (17) to get the following expressions:

$$
\begin{aligned}
r= & 1+3 H^{-2}\left(\frac{\frac{\rho_{0}}{3}(3 \xi+\gamma-3)(1+z)^{-(3 \xi+\gamma-3)} H-H^{3}\left(\frac{\omega v^{2} \gamma^{3}}{3}\right)}{2 H\left(1-\gamma-\frac{\omega}{6} v^{2} \gamma^{2}\right)-\frac{C^{2}}{4 \pi} H^{3}\left(H^{2}+\delta \pi\right)^{-2}\left(H^{2}+2 \delta \pi\right)}\right) \\
+ & H^{-3}\left(2 H\left(1-\gamma-\frac{\omega}{6} v^{2} \gamma^{2}\right)-\frac{2}{3} F H^{3}\left(H^{2}+\delta \pi\right)^{-2}\left(H^{2}+2 \delta \pi\right)\right)^{-1} \\
& \left(\frac{\rho_{0}}{3}(3 \xi+\gamma-3)^{2} H^{2}(1+z)^{-(3 \xi+\gamma-3)}-\frac{2 \omega}{3} H^{4} \gamma^{4} v^{2}\right. \\
+ & 2\left(\frac{\frac{\rho_{0}}{3}(3 \xi+\gamma-3)(1+z)^{-(3 \xi+\gamma-3)} H-H^{3}\left(\frac{\omega v^{2} \gamma^{3}}{3}\right)}{2 H\left(1-\gamma-\frac{\omega}{6} v^{2} \gamma^{2}\right)-\frac{C^{2}}{4 \pi} H^{3}\left(H^{2}+\delta \pi\right)^{-2}\left(H^{2}+2 \delta \pi\right)}\right)^{2} \\
& \left(F H^{2}\left(H^{2}+\delta \pi\right)^{-2}\left(H^{2}+2 \delta \pi\right)-\frac{4}{3} F H^{4}\left(H^{2}+\delta \pi\right)^{-3}\left(H^{2}+2 \delta \pi\right)\right.
\end{aligned}
$$




$$
\begin{aligned}
& \left.+\frac{2}{3} F H^{4}\left(H^{2}+\delta \pi\right)^{-2}-\left(1-\gamma-\frac{\omega}{6} v^{2} \gamma^{2}\right)\right) \\
& -\left(\frac{\frac{\rho_{0}}{3}(3 \xi+\gamma-3)(1+z)^{-(3 \xi+\gamma-3)} H-H^{3}\left(\frac{\omega v^{2} \gamma^{3}}{3}\right)}{2 H\left(1-\gamma-\frac{\omega}{6} v^{2} \gamma^{2}\right)-\frac{C^{2}}{4 \pi} H^{3}\left(H^{2}+\delta \pi\right)^{-2}\left(H^{2}+2 \delta \pi\right)}\right) \\
& \left.\left(\frac{2}{3} H^{2} \omega \gamma^{3} v^{2}-H^{2} \omega \gamma^{3} v^{2}-\frac{\rho_{0}}{3}(3 \xi+\gamma-3)^{2}(1+z)^{-(3 \xi+\gamma-3)}\right)\right), \\
& s=\frac{1}{3}\left(3 H^{-2}\left(\frac{\frac{\rho_{0}}{3}(3 \xi+\gamma-3)(1+z)^{-(3 \xi+\gamma-3)} H-H^{3}\left(\frac{\omega v^{2} \gamma^{3}}{3}\right)}{2 H\left(1-\gamma-\frac{\omega}{6} v^{2} \gamma^{2}\right)-\frac{C^{2}}{4 \pi} H^{3}\left(H^{2}+\delta \pi\right)^{-2}\left(H^{2}+2 \delta \pi\right)}\right)\right. \\
& +H^{-3}\left(2 H\left(1-\gamma-\frac{\omega}{6} v^{2} \gamma^{2}\right)-\frac{2}{3} F H^{3}\left(H^{2}+\delta \pi\right)^{-2}\left(H^{2}+2 \delta \pi\right)\right)^{-1} \\
& \left(\frac{\rho_{0}}{3}(3 \xi+\gamma-3)^{2} H^{2}(1+z)^{-(3 \xi+\gamma-3)}-\frac{2 \omega}{3} H^{4} \gamma^{4} v^{2}\right. \\
& +2\left(\frac{\frac{\rho_{0}}{3}(3 \xi+\gamma-3)(1+z)^{-(3 \xi+\gamma-3)} H-H^{3}\left(\frac{\omega v^{2} \gamma^{3}}{3}\right)}{2 H\left(1-\gamma-\frac{\omega}{6} v^{2} \gamma^{2}\right)-\frac{C^{2}}{4 \pi} H^{3}\left(H^{2}+\delta \pi\right)^{-2}\left(H^{2}+2 \delta \pi\right)}\right)^{2} \\
& \left(F H^{2}\left(H^{2}+\delta \pi\right)^{-2}\left(H^{2}+2 \delta \pi\right)-\frac{4}{3} F H^{4}\left(H^{2}+\delta \pi\right)^{-3}\left(H^{2}+2 \delta \pi\right)\right. \\
& \left.+\frac{2}{3} F H^{4}\left(H^{2}+\delta \pi\right)^{-2}-\left(1-\gamma-\frac{\omega}{6} v^{2} \gamma^{2}\right)\right) \\
& -\left(\frac{\frac{\rho_{0}}{3}(3 \xi+\gamma-3)(1+z)^{-(3 \xi+\gamma-3)} H-H^{3}\left(\frac{\omega v^{2} \gamma^{3}}{3}\right)}{2 H\left(1-\gamma-\frac{\omega}{6} v^{2} \gamma^{2}\right)-\frac{C^{2}}{4 \pi} H^{3}\left(H^{2}+\delta \pi\right)^{-2}\left(H^{2}+2 \delta \pi\right)}\right) \\
& \left.\left.\left(\frac{2}{3} H^{2} \omega \gamma^{3} v^{2}-H^{2} \omega \gamma^{3} v^{2}-\frac{\rho_{0}}{3}(3 \xi+\gamma-3)^{2}(1+z)^{-(3 \xi+\gamma-3)}\right)\right)\right) \\
& \left(-\frac{3}{2}-\frac{\rho_{0}(3 \xi+\gamma-3)(1+z)^{-(3 \xi+\gamma-3)} H-H^{3} \omega \gamma^{3} v^{2}}{6 H^{3}\left(1-\gamma-\frac{\omega}{6} v^{2} \gamma^{2}\right)-2 F H^{5}\left(H^{2}+\delta \pi\right)^{-2}\left(H^{2}+2 \delta \pi\right)}\right)^{-1} .
\end{aligned}
$$

Figure 11 gives plot of statefinder parameter $s$ against $r$ in the case of RHDE. It is clear from the plot that we have $r=1$ for $s=0$. This corresponds to the standard $\Lambda$ CDM cosmological model for accelerating universe.

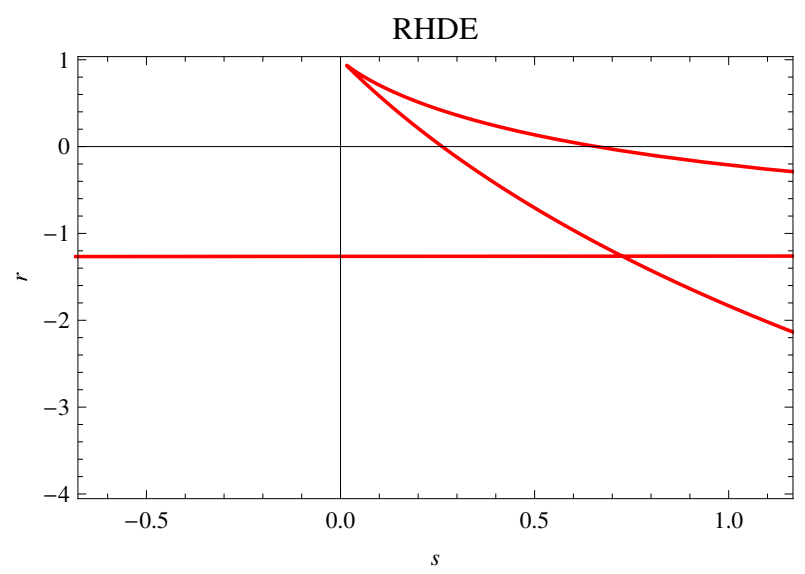

Figure 11. Statefinder parameter. 


\section{3. $S M H D E$}

For SMHDE, we customize Equations (37) and (38) using Equation (23) to attain the following expressions:

$$
\begin{aligned}
& r=1+3 H^{-2}\left(\frac{\rho_{0}}{3}(3 \xi+\gamma-3)(1+z)^{-(3 \xi+\gamma-3)} H-H^{3}\left(\frac{\omega v^{2} \gamma^{3}}{3}\right)\right)(2 H(1-\gamma \\
& \left.\left.-\frac{\omega}{6} v^{2} \gamma^{2}\right)-\frac{C^{2}}{8 \pi R}\left(4 H^{3}\left(1+\frac{\delta \pi}{H^{2}}\right)^{\beta}-1\right)-2 \delta \pi \beta H\left(1+\frac{\delta \pi}{H^{2}}\right)^{\beta-1}\right)^{-1}+H^{-3}(2 H(1 \\
& \left.\left.-\gamma-\frac{\omega}{6} \gamma^{2} v^{2}\right)-\frac{C^{2}}{8 \pi R}\left(4 H^{3}\left(\left(1+\frac{\delta \pi}{H^{2}}\right)^{\beta}-1\right)-2 \delta \beta H\left(1+\frac{\delta \pi}{H^{2}}\right)^{\beta-1}\right)\right)^{-1}\left(\frac{2}{3} \omega \gamma^{4}\right. \\
& v^{2} H^{4}+\frac{1}{3}\left(\rho _ { 0 } ( 3 \xi + \gamma - 3 ) ( 1 + z ) ^ { - ( 3 \xi + \gamma - 3 ) } \left(\frac{\rho_{0}}{3}(3 \xi+\gamma-3)(1+z)^{-(3 \xi+\gamma-3)} H\right.\right. \\
& \left.-H^{3}\left(\frac{\omega v^{2} \gamma^{3}}{3}\right)\right)\left(2 H\left(1-\gamma-\frac{\omega}{6} v^{2} \gamma^{2}\right)-\frac{C^{2}}{8 \pi R}\left(4 H^{3}\left(1+\frac{\delta \pi}{H^{2}}\right)^{\beta}-1\right)-2 \delta \pi \beta H\right. \\
& \left.\left.\left(1+\frac{\delta \pi}{H^{2}}\right)^{\beta-1}\right)^{-1}+\rho_{0}(3 \xi+\gamma-3)^{2} H^{2}(1+z)^{-(3 \xi+\gamma-3)}\right)-2\left(1-\gamma-\frac{\omega}{6} \gamma^{2} v^{2}\right) \\
& -\frac{C^{2}}{8 \pi R}\left(12 H^{2}\left(\left(1+\frac{\delta \pi}{H^{2}}\right)^{\beta}-1\right)-10 \delta \pi \beta\left(1+\frac{\delta \pi}{H^{2}}\right)^{\beta-1}+4 \delta^{2} \pi^{2} \beta(\beta-1)\left(1+\frac{\delta \pi}{H^{2}}\right)^{\beta-1}\right. \\
& \left.H^{-2}\right)\left(( \frac { \rho _ { 0 } } { 3 } ( 3 \xi + \gamma - 3 ) ( 1 + z ) ^ { - ( 3 \xi + \gamma - 3 ) } H - H ^ { 3 } ( \frac { \omega v ^ { 2 } \gamma ^ { 3 } } { 3 } ) ) \left(2 H \left(1-\gamma-\frac{\omega}{6} v^{2}\right.\right.\right. \\
& \left.\left.\left.\gamma^{2}\right)-\frac{C^{2}}{8 \pi R}\left(4 H^{3}\left(1+\frac{\delta \pi}{H^{2}}\right)^{\beta}-1\right)-2 \delta \pi \beta H\left(1+\frac{\delta \pi}{H^{2}}\right)^{\beta-1}\right)^{-1}\right)^{2}-\frac{5}{3} \omega \gamma^{3} v^{2} H^{2}\left(\frac{\rho_{0}}{3}\right. \\
& \left.(3 \xi+\gamma-3)(1+z)^{-(3 \xi+\gamma-3)} H-H^{3}\left(\frac{\omega v^{2} \gamma^{3}}{3}\right)\right)\left(2 H\left(1-\gamma-\frac{\omega}{6} v^{2} \gamma^{2}\right)-\frac{C^{2}}{8 \pi R}\right. \\
& \left.\left.\left(4 H^{3}\left(1+\frac{\delta \pi}{H^{2}}\right)^{\beta}-1\right)-2 \delta \pi \beta H\left(1+\frac{\delta \pi}{H^{2}}\right)^{\beta-1}\right)^{-1}\right), \\
& s=\frac{1}{3}\left(3 H ^ { - 2 } ( \frac { \rho _ { 0 } } { 3 } ( 3 \xi + \gamma - 3 ) ( 1 + z ) ^ { - ( 3 \xi + \gamma - 3 ) } H - H ^ { 3 } ( \frac { \omega v ^ { 2 } \gamma ^ { 3 } } { 3 } ) ) \left(2 H\left(1-\gamma-\frac{\omega}{6} v^{2} \gamma^{2}\right)\right.\right. \\
& \left.-\frac{C^{2}}{8 \pi R}\left(4 H^{3}\left(1+\frac{\delta \pi}{H^{2}}\right)^{\beta}-1\right)-2 \delta \pi \beta H\left(1+\frac{\delta \pi}{H^{2}}\right)^{\beta-1}\right)^{-1}+H^{-3}\left(2 H\left(1-\gamma-\frac{\omega}{6} \gamma^{2} v^{2}\right)\right. \\
& \left.-\frac{C^{2}}{8 \pi R}\left(4 H^{3}\left(\left(1+\frac{\delta \pi}{H^{2}}\right)^{\beta}-1\right)-2 \delta \beta H\left(1+\frac{\delta \pi}{H^{2}}\right)^{\beta-1}\right)\right)^{-1}\left(\frac{2}{3} \omega \gamma^{4} v^{2} H^{4}+\frac{1}{3}\left(\rho_{0}(3 \xi\right.\right. \\
& +\gamma-3)(1+z)^{-(3 \xi+\gamma-3)}\left(\frac{\rho_{0}}{3}(3 \xi+\gamma-3)(1+z)^{-(3 \xi+\gamma-3)} H-H^{3}\left(\frac{\omega \nu^{2} \gamma^{3}}{3}\right)\right) \\
& \left(2 H\left(1-\gamma-\frac{\omega}{6} v^{2} \gamma^{2}\right)-\frac{C^{2}}{8 \pi R}\left(4 H^{3}\left(1+\frac{\delta \pi}{H^{2}}\right)^{\beta}-1\right)-2 \delta \pi \beta H\left(1+\frac{\delta \pi}{H^{2}}\right)^{\beta-1}\right)^{-1} \\
& \left.+\rho_{0}(3 \xi+\gamma-3)^{2} H^{2}(1+z)^{-(3 \xi+\gamma-3)}\right)-2\left(1-\gamma-\frac{\omega}{6} \gamma^{2} v^{2}\right)-\frac{C^{2}}{8 \pi R} \\
& \left(12 H^{2}\left(\left(1+\frac{\delta \pi}{H^{2}}\right)^{\beta}-1\right)-10 \delta \pi \beta\left(1+\frac{\delta \pi}{H^{2}}\right)^{\beta-1}+4 \delta^{2} \pi^{2} \beta(\beta-1)\left(1+\frac{\delta \pi}{H^{2}}\right)^{\beta-1}\right. \\
& \left.H^{-2}\right)\left(( \frac { \rho _ { 0 } } { 3 } ( 3 \xi + \gamma - 3 ) ( 1 + z ) ^ { - ( 3 \xi + \gamma - 3 ) } H - H ^ { 3 } ( \frac { \omega v ^ { 2 } \gamma ^ { 3 } } { 3 } ) ) \left(2 H\left(1-\gamma-\frac{\omega}{6} v^{2} \gamma^{2}\right)\right.\right.
\end{aligned}
$$




$$
\begin{aligned}
&-\left.\left.\frac{C^{2}}{8 \pi R}\left(4 H^{3}\left(1+\frac{\delta \pi}{H^{2}}\right)^{\beta}-1\right)-2 \delta \pi \beta H\left(1+\frac{\delta \pi}{H^{2}}\right)^{\beta-1}\right)^{-1}\right)^{2}-\frac{5}{3} \omega \gamma^{3} v^{2} H^{2}\left(\frac{\rho_{0}}{3}\right. \\
&\left.(3 \xi+\gamma-3)(1+z)^{-(3 \xi+\gamma-3)} H-H^{3}\left(\frac{\omega \nu^{2} \gamma^{3}}{3}\right)\right)\left(2 H\left(1-\gamma-\frac{\omega}{6} v^{2} \gamma^{2}\right)-\frac{C^{2}}{8 \pi R}\right. \\
&\left.\left.\left.\left(4 H^{3}\left(1+\frac{\delta \pi}{H^{2}}\right)^{\beta}-1\right)-2 \delta \pi \beta H\left(1+\frac{\delta \pi}{H^{2}}\right)^{\beta-1}\right)^{-1}\right)\right)\left(-H^{-2}\left(\frac{\rho_{0}}{3}(3 \xi+\gamma-3)\right.\right. \\
&\left.(1+z)^{-(3 \xi+\gamma-3)} H-H^{3}\left(\frac{\omega v^{2} \gamma^{3}}{3}\right)\right)\left(2 H\left(1-\gamma-\frac{\omega}{6} v^{2} \gamma^{2}\right)-\left(\frac { C ^ { 2 } } { 8 \pi R } \left(4 H^{3}\right.\right.\right. \\
&\left.\left.\left.\left.\left(1+\frac{\delta \pi}{H^{2}}\right)^{\beta}-1\right)\right)-2 \delta \pi \beta H\left(1+\frac{\delta \pi}{H^{2}}\right)^{\beta-1}\right)^{-1}-\frac{3}{2}\right)^{-1} .
\end{aligned}
$$

The variation of statefinder parameter $s$ against $r$ in the case of SMHDE is given in Figure 12. This corresponds to the standard $\Lambda \mathrm{CDM}$ cosmological model for accelerating universe as it is clear from the plot that we have $r=1$ for $s=0$.

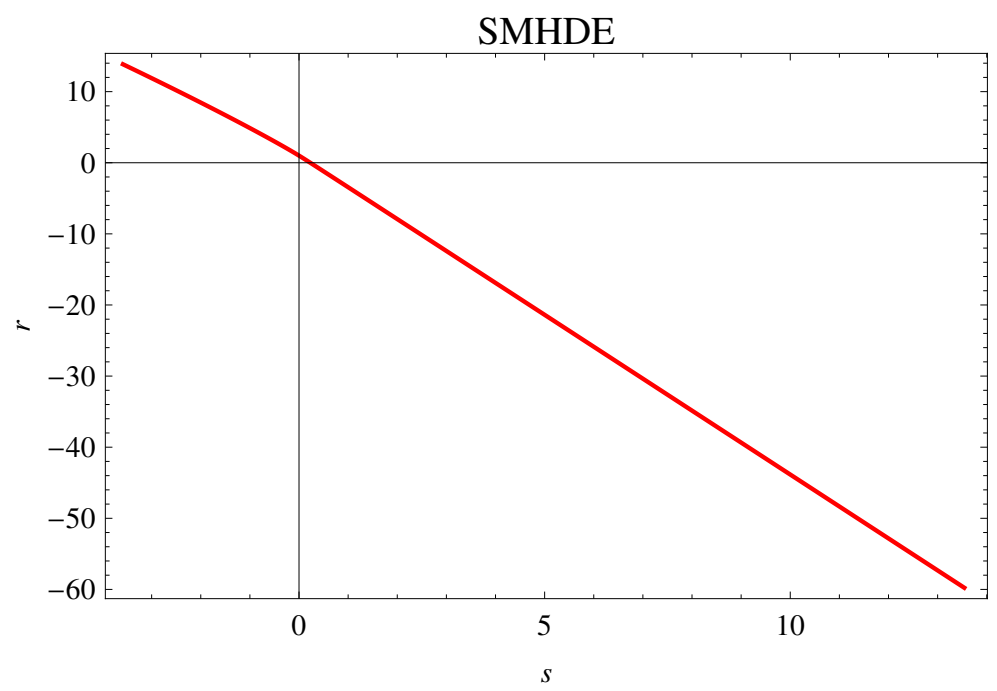

Figure 12. Statefinder parameter.

\section{Om Parameter}

Regarding observations, Om diagnostic appeared to be more simple as compared to $\{r, s\}$ statefinder. One among its salient features is its capability of discriminating dynamical DE models from $\Lambda$-CDM without any information about matter density. For $\Lambda$-CDM, quintessence and phantom models, Om has zero negative and positive curvatures, respectively.

Figure 13 gives the plot of Om-diagnostic for THDE versus redshift parameter $z$. Within the range $-0.9 \leq z \leq-0.1$, the trajectory shows positive curvature. This leads to phantom behavior of THDE model in the fractal universe. The plot of Om-diagnostic for RHDE against redshift parameter $z$ is given in Figure 14. For range $-0.9 \leq z \leq-0.1$, the plot represents positive curvature leading to phantom behavior of the RHDE model in the fractal universe. Following the same pattern, SMHDE also crosses the phantom line. It is evident in Figure 15, which shows positive curvature of trajectory. 
THDE

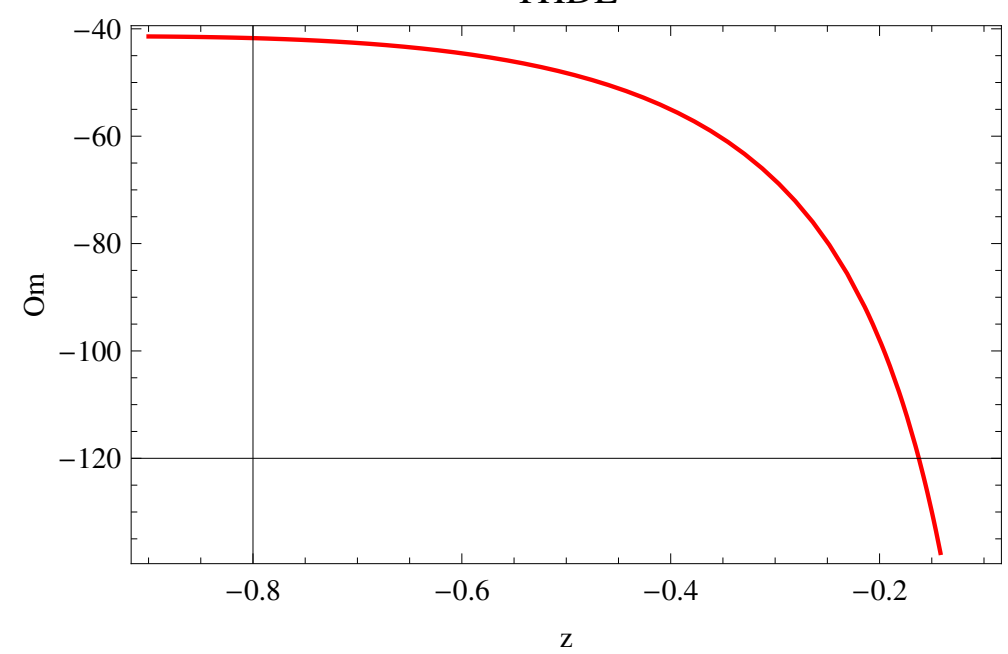

Figure 13. Om diagnostic parameter.

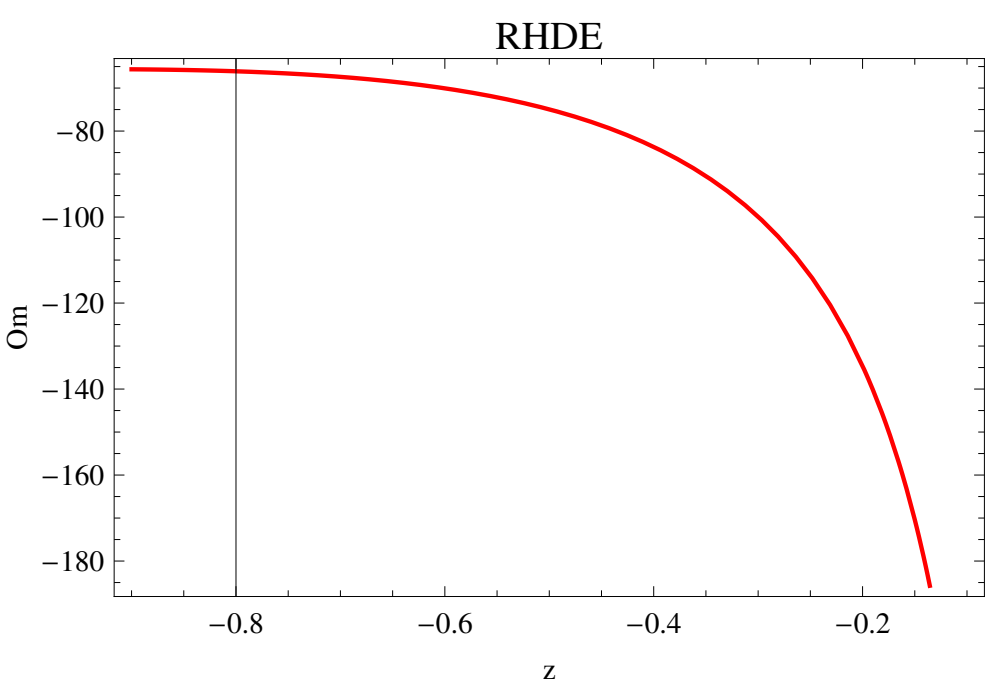

Figure 14. Om diagnostic parameter.

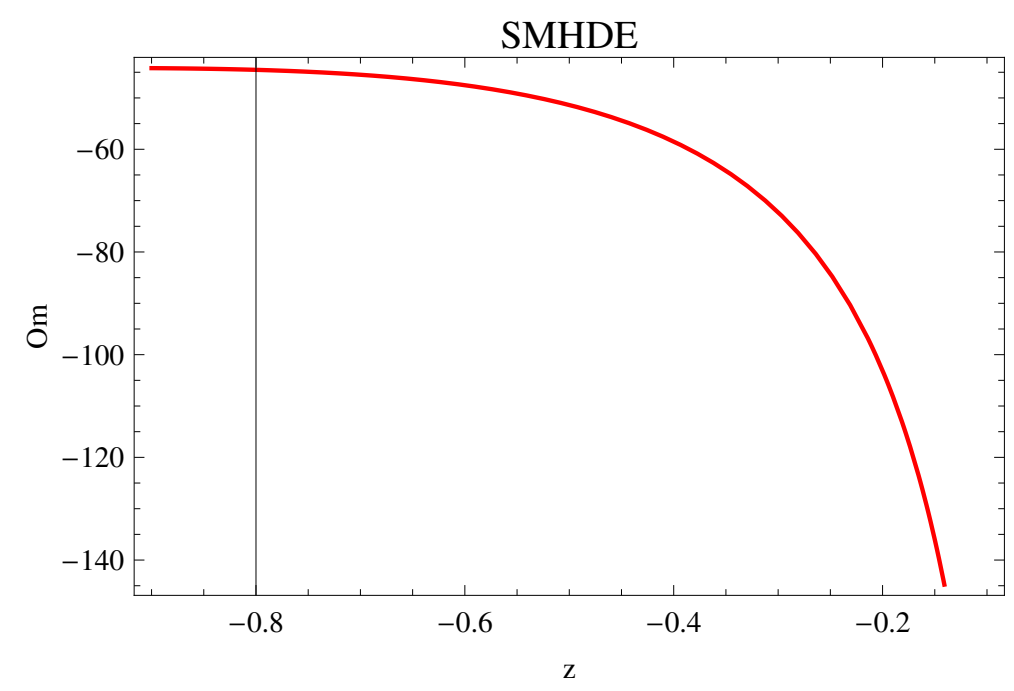

Figure 15. Om diagnostic parameter. 


\section{8. $\omega_{\text {eff }}{ }^{-} \omega_{e f f}^{\prime}$ Plane Analysis}

The dynamical property of DE models can be analyzed by using $\omega_{\text {eff }}-\omega_{\text {eff }}^{\prime}$ plane proposed by Linder and Caldwell [52]. Here, $\omega_{e f f}=\frac{p_{e f f}}{\rho_{e f f}}$ is the EoS parameter and $\omega_{e f f}^{\prime}$ is the evolution parameter for EoS parameter. To get this evolution parameter, we differentiate EoS parameter $\omega_{\text {eff }}$ with respect to $l n a$. The $\omega_{\text {eff }}-\omega_{\text {eff }}^{\prime}$ plane has been divided into two parts, namely "thawing" and "freezing". The conditions for thawing and freezing parts are $\omega_{e f f}<0, \omega_{e f f}^{\prime}>0$ and $\omega_{e f f}<0, \omega_{e f f}^{\prime}<0$, respectively.

\subsection{THDE}

By differentiating $\omega_{\text {eff }}$ for THDE with respect to $\ln a$, we get the following expression for $\omega_{e f f}^{\prime}$ in the case of THDE:

$$
\begin{aligned}
\omega_{\text {eff }}^{\prime}= & H^{-1}\left(\rho_{0}(1+z)^{-(3 \xi+\gamma-3)}+B H^{4-2 \delta}\right)^{-2}\left(\left(\rho_{0}(1+z)^{-(3 \xi+\gamma-3)}+B H^{4-2 \delta}\right)\right. \\
& \left(\frac{3 \xi \rho_{0}(3 \xi+\gamma-3)(1+z)^{-(3 \xi+\gamma-3)} H}{\gamma-3}-B(4-2 \delta) H^{3-2 \delta}\right. \\
& \left(\frac{\rho_{0}(3 \xi+\gamma-3)(1+z)^{-(3 \xi+\gamma-3)} H-H^{3} \omega \gamma^{3}(1+z)^{2 \gamma}\left(\gamma^{2}\right)}{6 H\left(1-\gamma-\frac{\omega}{6}(1+z)^{2 \gamma} \gamma^{2}\right)-B(4-2 \delta) H^{3-2 \delta}}\right)+\frac{B}{\gamma-3} \\
& (4-2 \delta)\left(H ^ { 2 - 2 \delta } \left(\left(\frac{\rho_{0}(3 \xi+\gamma-3)(1+z)^{-(3 \xi+\gamma-3)}}{3}-\frac{5 H^{2} \omega \gamma^{3} v^{2}}{3}\right)\right.\right. \\
& \left(\frac{\rho_{0}(3 \xi+\gamma-3)(1+z)^{-(3 \xi+\gamma-3)} H-H^{3} \omega \gamma^{3}(1+z)^{2 \gamma}\left(\gamma^{2}\right)}{6 H\left(1-\gamma-\frac{\omega}{6}(1+z)^{2 \gamma} \gamma^{2}\right)-B(4-2 \delta) H^{3-2 \delta}}\right) \\
+ & \frac{\rho_{0}(3 \xi+\gamma-3)(1+z)^{-(3 \xi+\gamma-3)} H^{2}}{3}+\left(\frac{B(4-2 \delta)(3-2 \delta) H^{2-2 \delta}}{3}-2(1-\gamma\right. \\
- & \left.\left.\frac{\omega}{6} v^{2} \gamma^{2}\right)\right)\left(\frac{\rho_{0}(3 \xi+\gamma-3)(1+z)^{-(3 \xi+\gamma-3)} H-H^{3} \omega \gamma^{3}(1+z)^{2 \gamma}\left(\gamma^{2}\right)}{6 H\left(1-\gamma-\frac{\omega}{6}(1+z)^{2 \gamma} \gamma^{2}\right)-B(4-2 \delta) H^{3-2 \delta}}\right) \\
& \left(2 H\left(1-\gamma-\frac{\omega}{6} v^{2} \gamma^{2}\right)-\frac{B}{3}(4-2 \delta) H^{3-2 \delta}\right)^{-1}+(2-2 \delta) H^{1-2 \delta} \\
& \left.\left(\frac{\rho_{0}(3 \xi+\gamma-3)(1+z)^{-(3 \xi+\gamma-3)} H-H^{3} \omega \gamma^{3}(1+z)^{2 \gamma}\left(\gamma^{2}\right)}{6 H\left(1-\gamma-\frac{\omega}{6}(1+z)^{2 \gamma} \gamma^{2}\right)-B(4-2 \delta) H^{3-2 \delta}}\right)\right) \\
- & \left(\frac{3 \xi \rho_{0}(1+z)^{-(3 \xi+\gamma-3)}}{\gamma-3}-B H^{4-2 \delta}+\frac{B(4-2 \delta) H^{2-2 \delta}}{\gamma-3}\right. \\
& \left.\left(\frac{\rho_{0}(3 \xi+\gamma-3)(1+z)^{-(3 \xi+\gamma-3)} H-H^{3} \omega \gamma^{3}(1+z)^{2 \gamma}\left(\gamma^{2}\right)}{6 H\left(1-\gamma-\frac{\omega}{6}(1+z)^{2 \gamma} \gamma^{2}\right)-B(4-2 \delta) H^{3-2 \delta}}\right)\right) \\
& \rho_{0}(3 \xi+\gamma-3)(1+z)^{-(3 \xi+\gamma-3)} H+B(4-2 \delta) H^{3-2 \delta} \\
& \left.\left(\frac{\rho_{0}(3 \xi+\gamma-3)(1+z)^{-(3 \xi+\gamma-3)} H-H^{3} \omega \gamma^{3}(1+z)^{2 \gamma}\left(\gamma^{2}\right)}{6 H\left(1-\gamma-\frac{\omega}{6}(1+z)^{2 \gamma} \gamma^{2}\right)-B(4-2 \delta) H^{3-2 \delta}}\right)\right) \\
& (3 \xi)
\end{aligned}
$$

In the case of THDE, the plot for $\omega_{e f f}-\omega_{e f f}^{\prime}$ is given by Figure 16. We use the same values of constant as described above. It is quite clear from the plot that the Eos parameter $\omega_{\text {eff }}<0$ and the evolutionary parameter $\omega_{e f f}^{\prime}<0$ as well. This shows THDE represents the freezing region. 


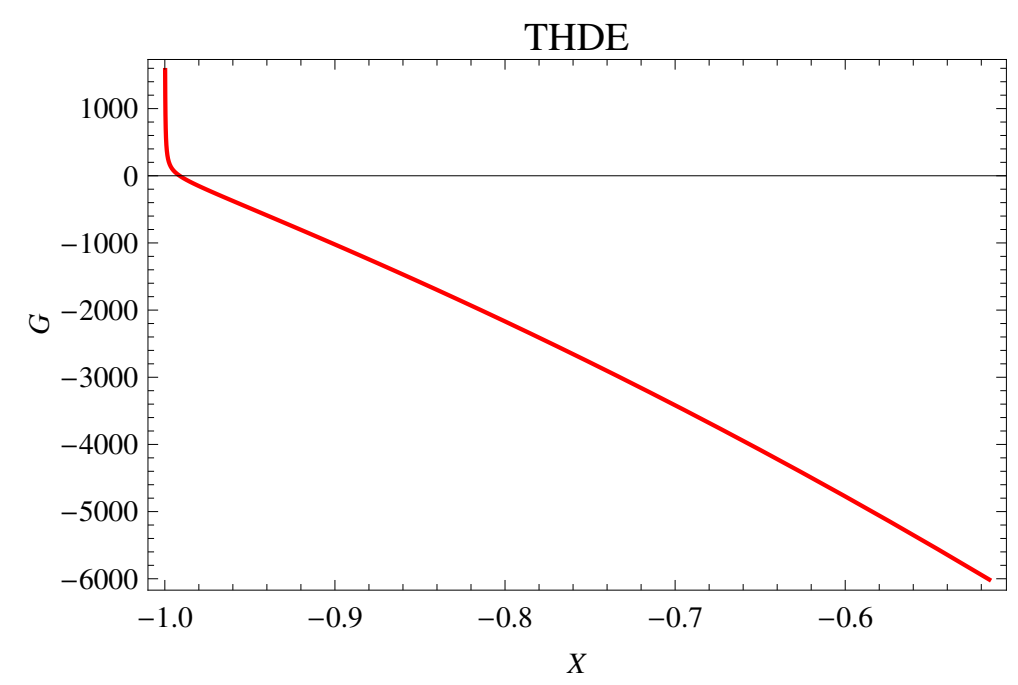

Figure 16. Plot of EoS parameter $\omega_{\text {eff }}=X$ and its evolution parameter $\omega_{\text {eff }}^{\prime}=G$.

8.2. $R H D E$

The expression for $\omega_{e f f}^{\prime}$ in the case of RHDE takes the following form:

$$
\begin{aligned}
& \omega_{\text {eff }}^{\prime}=H^{-1}\left(\rho_{0}(1+z)^{-(3 \xi+\gamma-3)}+F H^{2}\left(H^{2}+\delta \pi\right)^{-1}\right)^{-2}\left(\left(\rho_{0}(1+z)^{-(3 \xi+\gamma-3)}\right.\right. \\
& \left.+F H^{2}\left(H^{2}+\delta \pi\right)^{-1}\right)\left(\frac{3 \xi \rho_{0}(3 \xi+\gamma-3)(1+z)^{-(3 \xi+\gamma-3)} H}{(\gamma-3)}+\frac{H^{-1}}{(\gamma-3)} 2 F H^{3}\right. \\
& \left(H^{2}+\delta \pi\right)^{-2}\left(H^{2}+2 \delta \pi\right)\left(2 H\left(1-\gamma-\frac{\omega}{6} v^{2} \gamma^{2}\right)-\frac{2}{3} F H^{3}\left(H^{2}+\delta \pi\right)^{-2}\right. \\
& \left.\left(H^{2}+2 \delta \pi\right)\right)^{-1}\left(\frac{\rho_{0}}{3}(3 \xi+\gamma-3)^{2} H^{2}(1+z)^{-(3 \xi+\gamma-3)}-\frac{2 \omega}{3} H^{4} \gamma^{4} v^{2}\right. \\
& +2\left(\frac{\frac{\rho_{0}}{3}(3 \xi+\gamma-3)(1+z)^{-(3 \xi+\gamma-3)} H-H^{3}\left(\frac{\omega v^{2} \gamma^{3}}{3}\right)}{2 H\left(1-\gamma-\frac{\omega}{6} v^{2} \gamma^{2}\right)-\frac{C^{2}}{4 \pi} H^{3}\left(H^{2}+\delta \pi\right)^{-2}\left(H^{2}+2 \delta \pi\right)}\right)^{2} \\
& \left(F H^{2}\left(H^{2}+\delta \pi\right)^{-2}\left(H^{2}+2 \delta \pi\right)-\frac{4}{3} F H^{4}\left(H^{2}+\delta \pi\right)^{-3}\right. \\
& \left.\left(H^{2}+2 \delta \pi\right)+\frac{2}{3} F H^{4}\left(H^{2}+\delta \pi\right)^{-2}-\left(1-\gamma-\frac{\omega}{6} v^{2} \gamma^{2}\right)\right) \\
& -\left(\frac{\frac{\rho_{0}}{3}(3 \xi+\gamma-3)(1+z)^{-(3 \xi+\gamma-3)} H-H^{3}\left(\frac{\omega v^{2} \gamma^{3}}{3}\right)}{2 H\left(1-\gamma-\frac{\omega}{6} v^{2} \gamma^{2}\right)-\frac{C^{2}}{4 \pi} H^{3}\left(H^{2}+\delta \pi\right)^{-2}\left(H^{2}+2 \delta \pi\right)}\right) \\
& \left.\left(\frac{2}{3} H^{2} \omega \gamma^{3} v^{2}-H^{2} \omega \gamma^{3} v^{2}-\frac{\rho_{0}}{3}(3 \xi+\gamma-3)^{2}(1+z)^{-(3 \xi+\gamma-3)}\right)\right) \\
& +2 F\left(\frac{\frac{\rho_{0}}{3}(3 \xi+\gamma-3)(1+z)^{-(3 \xi+\gamma-3)} H-H^{3}\left(\frac{\omega v^{2} \gamma^{3}}{3}\right)}{2} H\left(1-\gamma-\frac{\omega}{6} v^{2} \gamma^{2}\right)\right. \\
& \left.-\frac{C^{2}}{4 \pi} H^{3}\left(H^{2}+\delta \pi\right)^{-2}\left(H^{2}+2 \delta \pi\right)\right)^{2}\left(3 H^{2}\left(H^{2}+\delta \pi\right)^{-2}\left(H^{2}+2 \delta \pi\right)\right. \\
& \left.-4 H^{4}\left(H^{2}+\delta \pi\right)^{-3}\left(H^{2}+2 \delta \pi\right)+2 H^{4}\left(H^{2}+\delta \pi\right)^{-2}\right)-2 F H^{3}\left(H^{2}+\delta \pi\right)^{-2} \\
& \left(H^{2}+2 \delta \pi\right)\left(\frac{\frac{\rho_{0}}{3}(3 \xi+\gamma-3)(1+z)^{-(3 \xi+\gamma-3)} H-H^{3}\left(\frac{\omega v^{2} \gamma^{3}}{3}\right)}{2 H\left(1-\gamma-\frac{\omega}{6} v^{2} \gamma^{2}\right)-\frac{C^{2}}{4 \pi} H^{3}\left(H^{2}+\delta \pi\right)^{-2}\left(H^{2}+2 \delta \pi\right)}\right)^{2}
\end{aligned}
$$




$$
\begin{aligned}
& \left.\left(\frac{1}{H^{2}(\gamma-3)} \frac{\frac{\rho_{0}}{3}(3 \xi+\gamma-3)(1+z)^{-(3 \xi+\gamma-3)} H-H^{3}\left(\frac{\omega v^{2} \gamma^{3}}{3}\right)}{2 H\left(1-\gamma-\frac{\omega}{6} v^{2} \gamma^{2}\right)-\frac{C^{2}}{4 \pi} H^{3}\left(H^{2}+\delta \pi\right)^{-2}\left(H^{2}+2 \delta \pi\right)}+1\right)\right) \\
+ & \frac{1}{H(\gamma-3)}\left(3 \xi H \rho_{0}(1+z)^{-(3 \xi+\gamma-3)}+H(3-\gamma) F H^{4}\left(H^{2}+\delta \pi\right)^{-1}\right. \\
+ & \frac{2}{3} F H^{3}\left(H^{2}+\delta \pi\right)^{-2}\left(H^{2}+2 \delta \pi\right)\left(\rho_{0}(3 \xi+\gamma-3)(1+z)^{-(3 \xi+\gamma-3)} H\right. \\
- & \left.\left.H^{3} \omega \gamma^{3} v^{2}\right)\left(2 H\left(1-\gamma-\frac{\omega}{6} v^{2} \gamma^{2}\right)-\frac{2}{3} F H^{3}\left(H^{2}+\delta \pi\right)^{-2}\left(H^{2}+2 \delta \pi\right)\right)^{-1}\right) \\
& \left(\rho_{0}(3 \xi+\gamma-3)(1+z)^{-(3 \xi+\gamma-3)} H-H^{3} \omega \gamma^{3} v^{2}\right)\left(2 H\left(1-\gamma-\frac{\omega}{6} v^{2} \gamma^{2}\right)\right. \\
- & \left.\left.\frac{2}{3} F H^{3}\left(H^{2}+\delta \pi\right)^{-2}\left(H^{2}+2 \delta \pi\right)\right)^{-1}\right) .
\end{aligned}
$$

The plot for $\omega_{\text {eff }}-\omega_{\text {eff }}^{\prime}$ in the case of RHDE is given by Figure 17. Clearly, it is representing freezing region, being Eos parameter $\omega_{\text {eff }}<0$ and the evolutionary parameter $\omega_{\text {eff }}^{\prime}<0$.

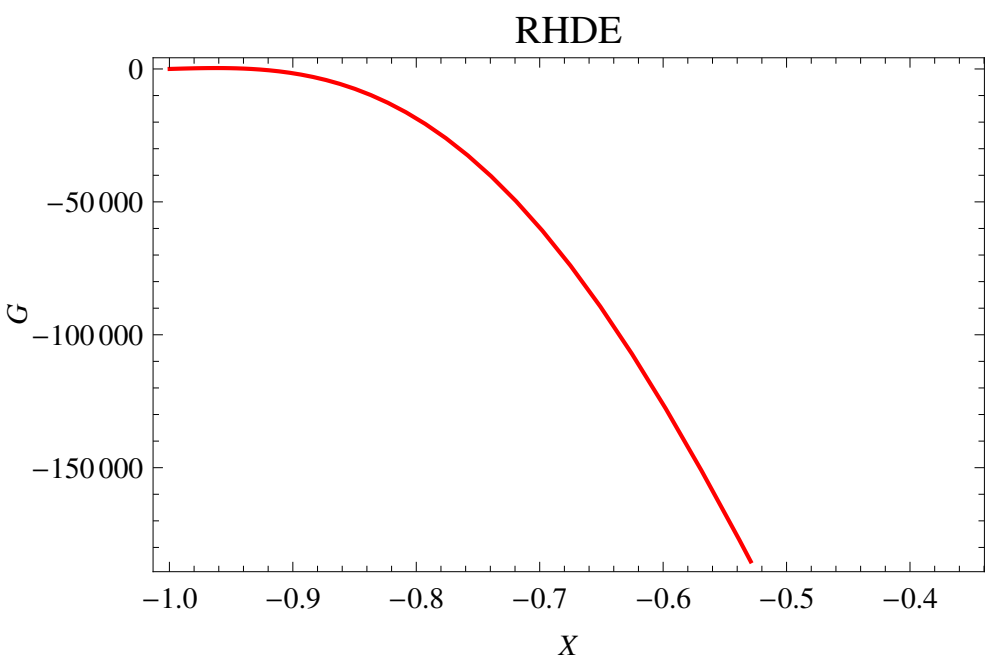

Figure 17. Plot of EoS parameter $\omega_{\text {eff }}=X$ and its evolution parameter $\omega_{\text {eff }}^{\prime}=G$.

\section{3. $S M H D E$}

For SMHDE, we get $\omega_{e f f}^{\prime}$ as given below:

$$
\begin{aligned}
\omega_{\text {eff }}^{\prime}= & H^{-1}\left(\frac{3 C^{2} H^{4}}{8 \pi R}\left(\left(1+\frac{\delta \pi}{H^{2}}\right)^{\beta}-1\right)+\rho_{0}(1+z)^{-(3 \xi+\gamma-3)}\right)^{-2}\left(\frac{3 C^{2} H^{4}}{8 \pi R}\left(\left(1+\frac{\delta \pi}{H^{2}}\right)^{\beta}-1\right)\right. \\
+ & \rho_{0}(1+z)^{-(3 \xi+\gamma-3)}\left(\frac { 1 } { 3 - \gamma } \left(-3 \xi \rho_{0}(3 \xi+\gamma-3)(1+z)^{-(3 \xi+\gamma-3)}-H^{-1} \frac{3 C^{2}}{8 \pi R}\left(12 H^{2}\right.\right.\right. \\
& \left(\left(1+\frac{\delta \pi}{H^{2}}\right)^{\beta}-1\right)-10 \delta \pi \beta\left(1+\frac{\delta \pi}{H^{2}}\right)^{\beta-1}+4 \delta^{2} \pi^{2} \beta(\beta-1)\left(1+\frac{\delta \pi}{H^{2}}\right)^{\beta-1} \\
& \left.H^{-2}\right)\left(( \frac { \rho _ { 0 } } { 3 } ( 3 \xi + \gamma - 3 ) ( 1 + z ) ^ { - ( 3 \xi + \gamma - 3 ) } H - H ^ { 3 } ( \frac { \omega v ^ { 2 } \gamma ^ { 3 } } { 3 } ) ) \left(\left(1-\gamma-\frac{\omega}{6} v^{2} \gamma^{2}\right)\right.\right. \\
& \left.\left.2 H-\frac{C^{2}}{8 \pi R}\left(4 H^{3}\left(1+\frac{\delta \pi}{H^{2}}\right)^{\beta}-1\right)-2 \delta \pi \beta H\left(1+\frac{\delta \pi}{H^{2}}\right)^{\beta-1}\right)^{-1}\right)^{3} \\
+ & \frac{3 C^{2}}{8 \pi R}\left(4 H^{3}\left(\left(1+\frac{\delta \pi}{H^{2}}\right)^{\beta}-1\right)-2 \delta \beta H\left(1+\frac{\delta \pi}{H^{2}}\right)^{\beta-1}\right)\left(2 H\left(1-\gamma-\frac{\omega}{6} \gamma^{2} v^{2}\right)\right.
\end{aligned}
$$




$$
\begin{aligned}
& \left.-\frac{C^{2}}{8 \pi R}\left(4 H^{3}\left(\left(1+\frac{\delta \pi}{H^{2}}\right)^{\beta}-1\right)-2 \delta \beta H\left(1+\frac{\delta \pi}{H^{2}}\right)^{\beta-1}\right)\right)^{-1}\left(\frac{2}{3} \omega \gamma^{4} v^{2} H^{4}\right. \\
& +\frac{1}{3}\left(\rho _ { 0 } ( 3 \xi + \gamma - 3 ) ( 1 + z ) ^ { - ( 3 \xi + \gamma - 3 ) } \left(\left(\frac{\rho_{0}}{3}(3 \xi+\gamma-3)(1+z)^{-(3 \xi+\gamma-3)} H\right.\right.\right. \\
& \left.-H^{3} \frac{\omega v^{2} \gamma^{3}}{3}\right)\left(2 H\left(1-\gamma-\frac{\omega}{6} v^{2} \gamma^{2}\right)-\frac{C^{2}}{8 \pi R}\left(4 H^{3}\left(1+\frac{\delta \pi}{H^{2}}\right)^{\beta}-1\right)-2 \delta \pi \beta H\right. \\
& \left.\left.\left.\left(1+\frac{\delta \pi}{H^{2}}\right)^{\beta-1}\right)^{-1}\right)+\rho_{0}(3 \xi+\gamma-3)^{2} H^{2}(1+z)^{-(3 \xi+\gamma-3)}\right)-2\left(1-\gamma-\frac{\omega}{6} \gamma^{2} v^{2}\right) \\
& -\frac{C^{2}}{8 \pi R}\left(\frac{\rho_{0}}{3}(3 \xi+\gamma-3)(1+z)^{-(3 \xi+\gamma-3)} H-H^{3}\left(\frac{\omega v^{2} \gamma^{3}}{3}\right)\right)\left(2 H\left(1-\gamma-\frac{\omega}{6} v^{2} \gamma^{2}\right)\right. \\
& \left.-\frac{C^{2}}{8 \pi R}\left(4 H^{3}\left(1+\frac{\delta \pi}{H^{2}}\right)^{\beta}-1\right)-2 \delta \pi \beta H\left(1+\frac{\delta \pi}{H^{2}}\right)^{\beta-1}\right)^{-1}\left(12 H^{2}\left(\left(1+\frac{\delta \pi}{H^{2}}\right)^{\beta}-1\right)\right. \\
& \left.-10 \delta \pi \beta\left(1+\frac{\delta \pi}{H^{2}}\right)^{\beta-1}+4 \delta^{2} \pi^{2} \beta(\beta-1)\left(1+\frac{\delta \pi}{H^{2}}\right)^{\beta-1} H^{-2}\right)\left(\left(\frac{\rho_{0}}{3}(3 \xi+\gamma-3)\right.\right. \\
& \left.(1+z)^{-(3 \xi+\gamma-3)} H-H^{3} \frac{\omega v^{2} \gamma^{3}}{3}\right)\left(2 H\left(1-\gamma-\frac{\omega}{6} v^{2} \gamma^{2}\right)-\frac{C^{2}}{8 \pi R}\left(4 H^{3}\left(1+\frac{\delta \pi}{H^{2}}\right)^{\beta}\right.\right. \\
& \text { - 1) } \left.\left.-2 \delta \pi \beta H\left(1+\frac{\delta \pi}{H^{2}}\right)^{\beta-1}\right)^{-1}\right)^{2}-\frac{5}{3} \omega \gamma^{3} v^{2} H^{2}\left(\frac{\rho_{0}}{3}(3 \xi+\gamma-3)(1+z)^{-(3 \xi+\gamma-3)} H\right. \\
& \left.-H^{3}\left(\frac{\omega v^{2} \gamma^{3}}{3}\right)\right)\left(2 H\left(1-\gamma-\frac{\omega}{6} v^{2} \gamma^{2}\right)-\frac{C^{2}}{8 \pi R}\left(4 H^{3}\left(1+\frac{\delta \pi}{H^{2}}\right)^{\beta}-1\right)-2 \delta \pi \beta H\right. \\
& \left.\left.\left(1+\frac{\delta \pi}{H^{2}}\right)^{\beta-1}\right)^{-1}\right)+\left(H^{-2}-3+\gamma\right) \frac{3 C^{2}}{8 \pi R}\left(4 H^{3}\left(\left(1+\frac{\delta \pi}{H^{2}}\right)^{\beta}-1\right)-2 \delta \beta H\right. \\
& \left.\left(1+\frac{\delta \pi}{H^{2}}\right)^{\beta-1}\right)\left(\frac{\rho_{0}}{3}(3 \xi+\gamma-3)(1+z)^{-(3 \xi+\gamma-3)} H-H^{3}\left(\frac{\omega v^{2} \gamma^{3}}{3}\right)\right)(2 H \\
& \left.\left.\left.\left(1-\gamma-\frac{\omega}{6} v^{2} \gamma^{2}\right)-\frac{C^{2}}{8 \pi R}\left(4 H^{3}\left(1+\frac{\delta \pi}{H^{2}}\right)^{\beta}-1\right)-2 \delta \pi \beta H\left(1+\frac{\delta \pi}{H^{2}}\right)^{\beta-1}\right)^{-1}\right)\right) \\
& -\frac{1}{H(3-\gamma)}\left(-3 \xi H \rho_{0}(1+z)^{-(3 \xi+\gamma-3)}-\frac{3 C^{2} H^{5}(3-\gamma)}{8 \pi R}\left(\left(1+\frac{\delta \pi}{H^{2}}\right)^{\beta}-1\right)\right. \\
& \left.-\frac{3 C^{2}}{8 \pi R}\left(4 H^{3}\left(\left(1+\frac{\delta \pi}{H^{2}}\right)^{\beta}-1\right)-2 \delta \pi \beta H\left(1+\frac{\delta \pi}{H^{2}}\right)^{\beta}\right)\right)\left(\frac{\rho_{0}}{3}(3 \xi+\gamma-3)\right. \\
& \left.(1+z)^{-(3 \xi+\gamma-3)} H-H^{3}\left(\frac{\omega v^{2} \gamma^{3}}{3}\right)\right)\left(2 H\left(1-\gamma-\frac{\omega}{6} v^{2} \gamma^{2}\right)-\left(\frac{C^{2}}{8 \pi R}\right.\right. \\
& \left.\left.\left(4 H^{3}\left(1+\frac{\delta \pi}{H^{2}}\right)^{\beta}-1\right)\right)-2 \delta \pi \beta H\left(1+\frac{\delta \pi}{H^{2}}\right)^{\beta-1}\right)^{-1} \frac{3 C^{2}}{8 \pi R}\left(4 H^{3}\left(\left(1+\frac{\delta \pi}{H^{2}}\right)^{\beta}-1\right)\right. \\
& \left.-2 \delta \beta H\left(1+\frac{\delta \pi}{H^{2}}\right)^{\beta-1}\right)\left(\frac{\rho_{0}}{3}(3 \xi+\gamma-3)(1+z)^{-(3 \xi+\gamma-3)} H-H^{3}\left(\frac{\omega v^{2} \gamma^{3}}{3}\right)\right)(2 H \\
& \left.\left.\left(1-\gamma-\frac{\omega}{6} v^{2} \gamma^{2}\right)-\frac{C^{2}}{8 \pi R}\left(4 H^{3}\left(1+\frac{\delta \pi}{H^{2}}\right)^{\beta}-1\right)-2 \delta \pi \beta H\left(1+\frac{\delta \pi}{H^{2}}\right)^{\beta-1}\right)^{-1}\right) \text {. }
\end{aligned}
$$

In the case of SMHDE, the plot for $\omega_{e f f}-\omega_{e f f}^{\prime}$ is given by Figure 18. Clearly, it is representing thawing region, being the EoS parameter $\omega_{e f f}<0$ and the evolutionary parameter $\omega_{e f f}^{\prime}>0$. 


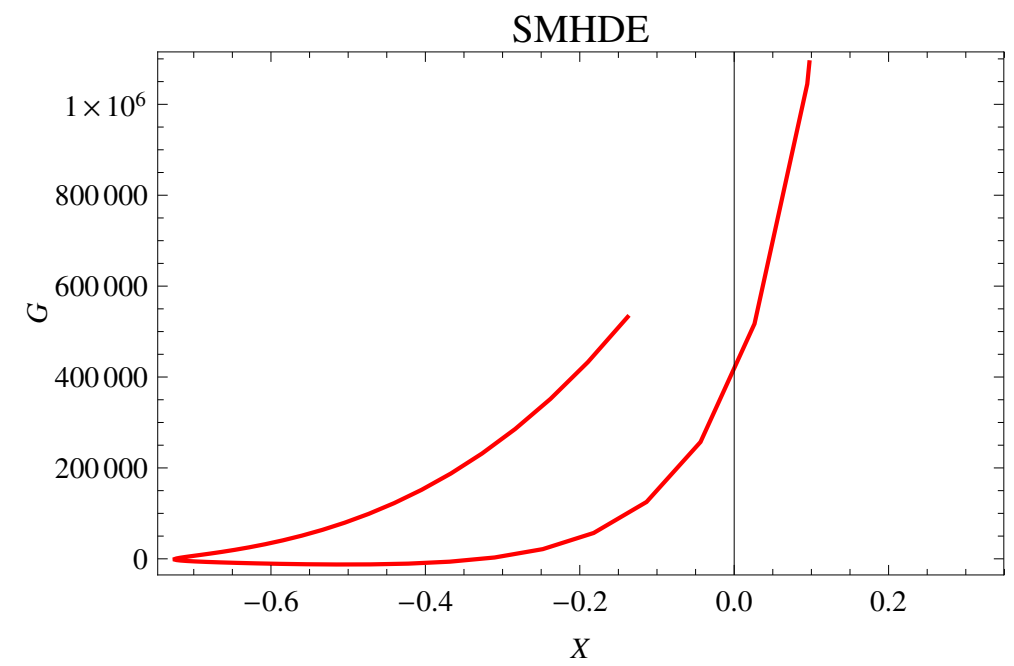

Figure 18. Plot of EoS parameter $\omega_{\text {eff }}=X$ and its evolution parameter $\omega_{\text {eff }}^{\prime}=G$.

\section{Conclusions}

In this paper, we have considered the flat FRW universe in fractal spacetime. We have investigated some cosmological implications such as EoS parameter, deceleration parameter, Om parameter, State-finder parameter and $\omega_{\text {eff }}-\omega_{\text {eff }}^{\prime}$ plane. For this purpose we have taken three DE models, namely Tsallis DE, Renyi DE and Sharma mittal DE. We have selected an appropriate interacting situation for HDE and DM to avoid coincidence. We have derived the expressions for all above-mentioned cosmological parameters in terms of red shift parameter $z$. We have fixed the constants as $\xi=-2$, $\rho=1, \delta=1, B=1, \gamma=2$ and $\alpha=1$ generally. For some cases of RHDE and SMHDE, we have taken $\xi=-2$ and $\beta=-800$. The deceleration parameter $q$ describes the evolution of the universe. The three cosmological models, i.e., THDE, RHDE and SMHDE, of the evolving universe transit from early decelerating phase $(q>0)$ to current accelerating phase $(q<0)$. On the basis of the EoS parameter $\omega_{\text {eff }}$, we can say THDE exhibit quintom-like nature as it shows transition of the universe from Cosmological constant towards quintessence. RHDE shows conversion of the universe from Cosmological constant towards quintessence. SMHDE shows quintom-like behavior. For the stability analysis of THDE, RHDE and SMHDE, we investigate the graphical behavior of square speed of sound. All three interacting HDE models under consideration in fractal universe are stable. From $\omega_{\text {eff }}-\omega_{\text {eff }}^{\prime}$ plane, we can say that THDE and RHDE represent the freezing region and reinforce the accelerated expansion of the universe, whereas the third model, SMHDE, represents the thawing region. In the same manner, we have $\{r, s\}=\{1,0\}$ representing standard $\Lambda C D M$ cosmological model of the accelerating universe for THDE, RHDE and SMHDE. The plots for Om diagnostic of THDE, RHDE and SMHDE are almost identical. The plot shows positive curvature leading to phantom behavior of selected HDE models. It is noted that the constraints on effective EoS are also compatible with recent results [53].

Finally, it is observed that EoS parameter of all DE models lies within the following constraints at the present epoch (Table 1). 
Table 1. Summary of the observational data on $\omega_{D E}$.

\begin{tabular}{ccc}
\hline$\omega_{\text {eff }}$ & Observational Schemes & Refrences \\
\hline$-1.13_{-0.25}^{+0.24}$ & Planck+WP+BAO & {$[54]$} \\
\hline$-1.09 \pm 0.17$ & Planck+WP+Union 2.1 & {$[54]$} \\
\hline$-1.13_{-0.14}^{+0.13}$ & Planck+WP+SNLS & {$[54]$} \\
\hline$-1.24_{-0.19}^{+0.18}$ & WMAP+eCMB+BAO+H + SNe Ia & {$[54]$} \\
\hline$-1.073_{-0.089}^{+0.090}$ & WMAP+eCMB+BAO+H & {$[55]$} \\
\hline$-1.084 \pm 0.063$ & WMAP+eCMB+BAO+H $+\mathrm{SNe}$ & {$[55]$} \\
\hline
\end{tabular}

Author Contributions: M.U.S. has done analysis as well as proof reading of the manuscript, A.I. has completed the manuscript with calculations and figures while A.J. has contributed in the improvement of the manuscript and prepared the final draft.

Funding: This research received no external funding.

Acknowledgments: Abdul Jawad is thankful to HEC, Islamabad, Pakistan for its financial support under the grant No: 5412/Federal/NRPU/R\&D/HEC/2016.

Conflicts of Interest: The authors declare no conflict of interest.

\section{References}

1. Peebles, P.J.E. Principles of Physical Cosmology; Princeton University Press: Princeton, NJ, USA, 1993.

2. Davis, M. Is the Universe Homogeneous on Large Scales? In Critical Dialogues in Cosmology; Turok, N., Ed.; World Scientific Publishing Company: Singapore, 1997; p. 13.

3. Wu, K.K.S.; Lahav, O.; Rees, M. The Large-Scale Smoothness of the Universe. Nature 1999, 397, 225. [CrossRef]

4. Joyce, M.; Montuori, M.; Sylos, L.F.; Pietronero, L. Comment on the paper "the eso slice project galaxy redshift survey: v. evidence for a d=3 sample dimensionality". Astron. Astrophys. 1999, 344, 387.

5. Coles, P. Cosmology: An unprincipled Universe? Nature 1998, 391, 120. [CrossRef]

6. Smoot, G.; Bennett, C.L.; Kogut, A.; Wright, E.L.; Aymon, J.; Boggess, N.W.; Cheng, E.S.; de Amici, G.; Gulkis, S.; Hauser, M.G.; et al. Structure in the COBE differential microwave radiometer first-year maps. Astrophys. J. 1992, 396, L1. [CrossRef]

7. Joyce, M.; Anderson, P.W.; Montuori, M.; Sylos Labini, F.; Pietronero, L. Fractal Cosmology in an Open Universe. Europhys. Lett. 2000, 49, 416. [CrossRef]

8. Mandelbrot, B. The Fractal Geometry of Nature. Am. J. Phys. 1983, 51, 286. [CrossRef]

9. Mandelbrot, B.B. Fractality, Lacunarity, and the Near-Isotropic Distribution of Galaxies. In Current Topics in Astrofundamental Physics: Primordial Cosmology; Sanchez, N., Zichichi, A., Eds.; NATO ASI Series (Series C: Mathematical and Physical Sciences); Springer: Dordrecht, The Netherlands, 1998; Volume 511, p. 583.

10. Coleman, P.H.; Pietronero, L. The fractal structure of the universe. Phys. Rep. 1992, 213, 311. [CrossRef]

11. Perlmutter, S.; Aldering, G.; Goldhaber, G.; Knop, R.A.; Nugent, P.; Castro, P.G.; Deustua, S.; Fabbro, S.; Goobar, A.; Groom, D.E.; et al. Measurements of $\Omega$ and $\Lambda$ from 42 high-redshift supernovae. Astrophys. J. 1999, 517, 565. [CrossRef]

12. Abazajian, K.; Adelman-McCarthy, J.K.; Ag'ueros, M.A.; Allam, S.S.; Anderson, K.S.; Anderson, S.F.; Annis, J.; Bahcall, N.A.; Baldry, I.K.; Bastian, S.; et al. The second data release of the sloan digital sky survey. Astron. J. 2004, 128, 502. [CrossRef]

13. Riess, A.G.; Filippenko, A.V.; Challis, P.; Clocchiatti, A.; Diercks, A.; Garnavich, P.M.; Gilliland, R.L.; Hogan, C.J.; Jha, S.; Kirshner, R.P.; et al. Observational evidence from supernovae for an accelerating universe and a cosmological constant. Astron. J. 1998, 116, 1009. [CrossRef]

14. Bamba, K.; Capozziello, S.; Nojiri, S.; Odintsov, S.D. Dark energy cosmology: the equivalent description via different theoretical models and cosmography tests. Astro. Phys. Space Sci. 2012, 342, 155-228. [CrossRef]

15. Hooft, G. Dimensional Reduction in Quantum Gravity. arXiv 2009, arXiv:gr-qc/9310026v2.

16. Susskind, L. The World as a Hologram. J. Math. Phys. 1995, 36, 6377. [CrossRef] 
17. Cohen, A.; Kaplan, D.; Nelson, A. Effective Field Theory, Black Holes, and the Cosmological Constant. Phys. Rev. Lett. 1999, 82, 4971. [CrossRef]

18. Li, M. A Model of Holographic Dark Energy. Phys. Lett. B 2004, 603 1. [CrossRef]

19. Fischler, W.; Susskind, L. Holography and Cosmology. arXiv 1998, arXiv:hep-th/9806039.

20. Nojiri, S.; Odintsov, S.D. Unifying phantom inflation with late-time acceleration: Scalar phantom non-phantom transition model and generalized holographic dark energy. Gen. Relativ. Gravit. 2006, 38, 1285-1304. [CrossRef]

21. Nojiri, S.; Odintsov, S.D. Inhomogeneous equation of state of the universe: Phantom era, future singularity, and crossing the phantom barrier. Phys. Rev. D 2005, 72, 023003. [CrossRef]

22. Sheykhi, A. Holographic scalar field models of dark energy. Phys. Rev. D 2011, 84, 107302. [CrossRef]

23. Hu, B.; Ling, Y. Interacting dark energy, holographic principle, and coincidence problem. Phys. Rev. D 2006, 73, 123510. [CrossRef]

24. Ma, Y.Z.; Gong, Y.; Chen, X. Features of holographic dark energy under combined cosmological constraints. Eur. Phys. J. C 2009, 60, 303. [CrossRef]

25. Masi, M. A step beyond Tsallis and Renyi entropies. Phys. Lett. A 2005, 338, 217. [CrossRef]

26. Tsallis, C. The Nonadditive Entropy Sq and Its Applications in Physics and Elsewhere: Some Remarks. Entropy 2011, 13, 1765. [CrossRef]

27. Renyi, A. Probability Theory; North-Holland: Amsterdam, The Netherlands, 1970.

28. Tsallis, C. Possible generalization of Boltzmann-Gibbs statistics. J. Stat. Phys. 1988, 52, 479. [CrossRef]

29. Jahromi, A.S.; Moosavi, S.A.; Moradpour, H.; Graça, J.M.; Lobo, I.P.; Salako, I.G.; Jawad, A. Generalized entropy formalism and a new holographic dark energy model. J. Phys. Lett. B. 2018, 2, 52.

30. Nojiri, S.; Odintsov, S.D.; Saridakis, E.N. Modified cosmology from extended entropy with varying exponent. Eur. Phys. J. C 2019, 79, 242. [CrossRef]

31. Calcagni, G. Quantum field theory, gravity and cosmology in a fractal universe. JHEP 2010, $1003,120$. [CrossRef]

32. Calcagni, G. Fractal universe and quantum gravity. Phys. Rev. Lett. 2010, 104, 251301. [CrossRef]

33. Calcagni, G. Multi-scale gravity and cosmology. JCAP 2013, 12, 41. [CrossRef]

34. Salvatelli, V.; Said, N.; Bruni, M.; Melchiorri, A.; Wands, D. Indications of a Late-Time Interaction in the Dark Sector. Phys. Rev. Lett. 2014, 113, 181301. [CrossRef]

35. Yang, T.; Guo, Z.K. Reconstructing the interaction between dark energy and dark matter using Gaussian processes. Phys. Rev. D 2015, 91, 123533. [CrossRef]

36. Feng, C.; Wang, B.; Su, R.-K. Testing the viability of the interacting holographic dark energy model by using combined observational constraints. JCAP 2007, 9, 005. [CrossRef]

37. Zimdahl, W.; Pavon, D.; Chimento, L.P. Interacting Quintessence. Phys. Lett. B 2001, 521, 133. [CrossRef]

38. Wang, B.; Zang, J.; Lin, C.-Y.; Abdalla, E.; Micheletti, S. Interacting dark energy and dark matter: Observational constraints from cosmological parameters. Nucl. Phys. B 2007, 778, 69-84. [CrossRef]

39. Amendola, L.; Tsujikawa, S.; Sami, M. Phantom damping of matter perturbations. Phys. Lett. B 2006, 632, 155-158. [CrossRef]

40. Ferreira, E.G.M.; Quintin, J.; Costa, A.A.; Abdalla, E.; Wang, B. Evidence for interacting dark energy from BOSS. arXiv 2017, arXiv:1412.2777.

41. Gupta, G.; Saridakis, E.N.; Sen, A.A. Nonminimal quintessence and phantom with nearly flat potentials. Phys. Rev. D 2009, 79, 123013. [CrossRef]

42. Santos, L. Constraining interacting dark energy with CMB and BAO future surveys. Phys. Rev. D 2017, 96, 103529. [CrossRef]

43. Tsallis, C.; Cirto, L.J.L. Black hole thermodynamical entropy. Eur. Phys. J. C 2013, 73, 2487. [CrossRef]

44. Tavayef, M.; Sheykhi, A.; Bamba, K.; Moradpour, H. Tsallis Holographic Dark Energy. arXiv 2018, arXiv:1804.02983v1.

45. Majhi, A. Non-extensive Statistical Mechanics and Black Hole Entropy From Quantum Geometry. Phys. Lett. B 2017, 775, 32-36. [CrossRef]

46. Komatsu, N. Cosmological model from the holographic equipartition law with a modified Rényi entropy. Eur. Phys. J. C 2017, 77, 229. [CrossRef]

47. Moradpour, H.; Bonilla, A.; Abreu, E.M.C.; Neto, J.A. Accelerated cosmos in a nonextensive setup. Phys. Rev. D 2017, 96, 123504. [CrossRef] 
48. Czinner, V.G.; Iguchi, H. Black hole entropy and the zeroth law of thermodynamics. Int. J. Mod. Phys. D 2015, 24, 1542015. [CrossRef]

49. Sharma, B.D.; Mitta, P. New nonadditive measures of entropy for discrete probability distributions. J. Math. Sci. 1975, 10, 28-40.

50. Sharma, B.D.; Mitta, P. New non-additive measures of relative information. J. Cmbin Inform. Syst. Sci. 1977, 2, 122-132.

51. Sahni, V. Statefinder-A new geometrical diagnostic of dark energy. JETP Lett. 2003, 77, 201-206. [CrossRef]

52. Caldwell, R.R.; Linder, E.V. The Limits of Quintessence. Phys. Rev. Lett. 2005, 95, 141301. [CrossRef]

53. Aviles, A.; Gruber, C.; Luongo, O.; Quevedo, H. Cosmography and constraints on the equation of state of the Universe in various parametrizations. Phys. Rev. D 2012, 86, 123516. [CrossRef]

54. Ade, P.A.R. Planck 2015 results. XIII. Cosmological parameters. AEA 2016, 594, A13.

55. Hinshaw, G.; Larson, D.; Komatsu, E.; Spergel, D.N.; Bennett, C.L.; Dunkley, J.; Nolta, M.R.; Halpern, M.; Hill, R.S.; Odegard, N.; et al. Nine-year Wilkinson Microwave Anisotropy Probe (WMAP) Observations: Cosmological Parameter Results. Astrophys. J. Suppl. 2013, 208, 19. [CrossRef]

(C) 2019 by the authors. Licensee MDPI, Basel, Switzerland. This article is an open access article distributed under the terms and conditions of the Creative Commons Attribution (CC BY) license (http:/ / creativecommons.org/licenses/by/4.0/). 